\title{
UN MINIATORE NELLA BOTTEGA DEGLI ASTRAPAS? ALCUNE OSSERVAZIONI ATTORNO ALLE IMMAGINI DEL TOLOMEO MARCIANO GR. Z. 516 (904).
}

\section{FRANCESCO LOVINO}

UDC: $75.057(495.622) " 09 "$

Original scientific paper

Manuscript received: 01. 11. 2015.

Revised manuscript accepted: 17. 03. 2016.

DOI: 10.1484/J.HAM.5.111358
F. Lovino

Institute of Art History

Academy of Science of the Czech Republic

Husova, 4

110 oo Prague, Czech Republic

lovino@udu.cas.cz

Between the end of the thirteenth century and the beginning of the fourteenth. Thessaloniki was a flourishing and cosmopolitan city: in this context was realized the Ptolemy Marc. gr. Z. 516 (904), then belonging to cardinal Basilios Bessarion and today preserved in the Marciana Library of Venice. Copied in Greek by a Frank named Andrea Teluntàs, the manuscript contained also a cryptic cycle of illumination that once Italo Furlan defined as an ethic and philosophical Christian Weltanschaung.

The paper focuses on the illuminated cycle contained in the Ptolemy Marc. gr. Z. 516 (904), analyzing its artistic and literary sources and providing also a stylistic reading of it.

Keywords: Astrapas, Marc. gr. Z. 516 (904), Thessaloniki, Claudius Ptolemy, Gračanica, Paleologan Illumination, Stefan Uroš II Milutin, Aristotelianism

«Ogni uomo per natura desidera conoscere» Aristotele

\section{PREMESSA.}

In uno studio dedicato all'arte della memoria di Raimondo Lullo pubblicato nel 1954, la studiosa inglese Frances A. Yates scriveva che l'articolo in questione non conteneva alcuna conclusione, poiché si trattava non di una fine ma di un principio ${ }^{1}$. Allo stesso modo, il repertorio di immagini contenuto nel manoscritto Marc. gr. Z. 516 (904), oggetto delle seguenti pagine, rappresenta ancora un percorso inesplorato per scavare le ispirazioni e le aspirazioni culturali degli intellettuali bizantini dell'età paleologa. Alcuni aspetti sono stati svelati nei precedenti studi di Elpidio Mioni e Italo Furlan ${ }^{2}$; l'intento quest'oggi è di spingere qualche centimetro più in là il tentativo di illuminare ulteriori fonti letterarie e $i$ possibili modelli artistici che hanno guidato la creazione di un codice che Furlan ha definito "Weltanschauung cristiana di carattere etico-filosofico"3.

\section{IL MANOSCRITTO.}

Il Marc. gr. Z. 516 (904) appare citato per la prima volta al numero 266 dell'inventario della donazione del cardinal Bessarione $^{4}$, che riproduce a sua volta l'ex libris bessarioneo al f. $5 \mathrm{r}^{5}$. Cartaceo (mm 305X220, ff. I+208), il codice contiene il testo acefalo della Geografia di Tolomeo, corredata da numerose carte geografiche tratte da Agatodemone di Alessandria (ff. 5r-139v), la Tacticta theoria, trattato militare di Eliano (ff. 142r-156r), e due scritti di Erone di Alessandria, gli Pneumatica in due libri e gli Automata (ff. 16or-208v). L'intero codice, ad eccezione di alcune integrazioni dovute a copisti anonimi, si deve ad Andrea Teluntàs, figlio di Franco e originario di Nauplia, nel Pelopponeso, come riporta la sottoscrizione al f. 208v, scritta con grafia inelegante e con numerosi errori di itacismo e ortografia ${ }^{6}$. Le difficoltà ortografiche di Teluntàs, rimarcate da Mioni, suggeriscono che il copista potesse non essere di natali grecofoni: Nauplia fu feudo franco dal 1210

\footnotetext{
' F. YATES, Lull's Theory of the Elements, The Journal of Courtauld and Warburg Institutes, 17, 1954, p. 173.

${ }^{2}$ I. FURLAN, Codici greci illustrati della Biblioteca Marciana. IV, Milano 1981, p. 30-48; E. MIONI, Le tavole aggiunte alla Geografia di Tolomeo nel cod. Marc. gr. 516, in Studi bizantini e neogreci, (Università degli studi di Lecce, Facoltà di Lettere e Filosofia, Istituto di Storia Medioevale e Moderna. Saggi e Ricerche. 7), Atti del IV congresso nazionale di studi bizantini, (Lecce e Calimera, 21-24 aprile 1980), P. L. Leone (dir.), Galatina 1983, p. 57-67; I. FURLAN, Il libro enciclopedico di maestro Astrapas, in L'arte di Bisanzio e l'Italia al tempo dei Paleologi 1261-1453, A. Iacobini e M. Della Valle (dir.), Roma 1999, pp. 113-123. ${ }^{3}$ I. FURLAN, Il libro op. cit. (n. 2), p. 62

${ }^{4}$ Scrive Mioni che Bessarione commissionò al fido Giovanni Roso una copia del codice, l'attuale Marc. gr. Z 388 (833). E. MIONI, Bessarione scriba e alcuni suoi collaboratori, in Miscellanea marciana di studi bessarionei (a coronamento del V centenario della donazione nicena), Padova 1976, p. 302-304. Schnabel ritiene invece che la copia del Roso derivi da un esemplare della tradizione testuale A, forse il Vat. gr. 177 appartenuto a Planude, mentre il Tolomeo marciano gr. Z. 516 (904) appartiene alla tradizione testuale R: P. SCHNABEL, Text und Karten des Ptolämeus, Leipzig 1938, p. 23; S. BERNARDINELLO, Le carte dell'Africa nella Geografia di Tolomeo: lettura dal codice Marciano gr. Z. 516, in Atti e memorie dell'Accademia Patavina di Scienze, Lettere e Arti, 109, 1996-1997, p. 55.

${ }^{5}$ Edito in H. OMONT, Inventaire des manuscrits grecs et latins donnés à Saint-Marc de Venise par le Cardinal Bessarion (1468), Revue des bibliothèques, IV, 1894, p. 129-187.

${ }^{6}$ E. MIONI, op. cit. (n. 2), 1983, p. 57. Il fatto che un singolo scriba si occupò interamente di copiare il Marc. gr. Z. 516 (904) è degno di nota: in età paleologa infatti la diffusione dei codici miscellanei avveniva perlopiù attraverso il lavoro di un'equipe di copisti, coordinati da un unico maestro che sceglieva i testi e assegnava i compiti agli scribi. Sull'argomento si veda D. BIANCONI, Libri e mani. Sulla formazione di alcune miscellanee dell'età dei Paleologhi, "Segno e Testo", 2, 2004, (= Il codice miscellaneo. Tipologie e funzioni, Atti del Convegno internazionale (Cassino, 14-17 maggio 2003), E. Crisci e O. Pecere (dir.), p. 311-363.
} 


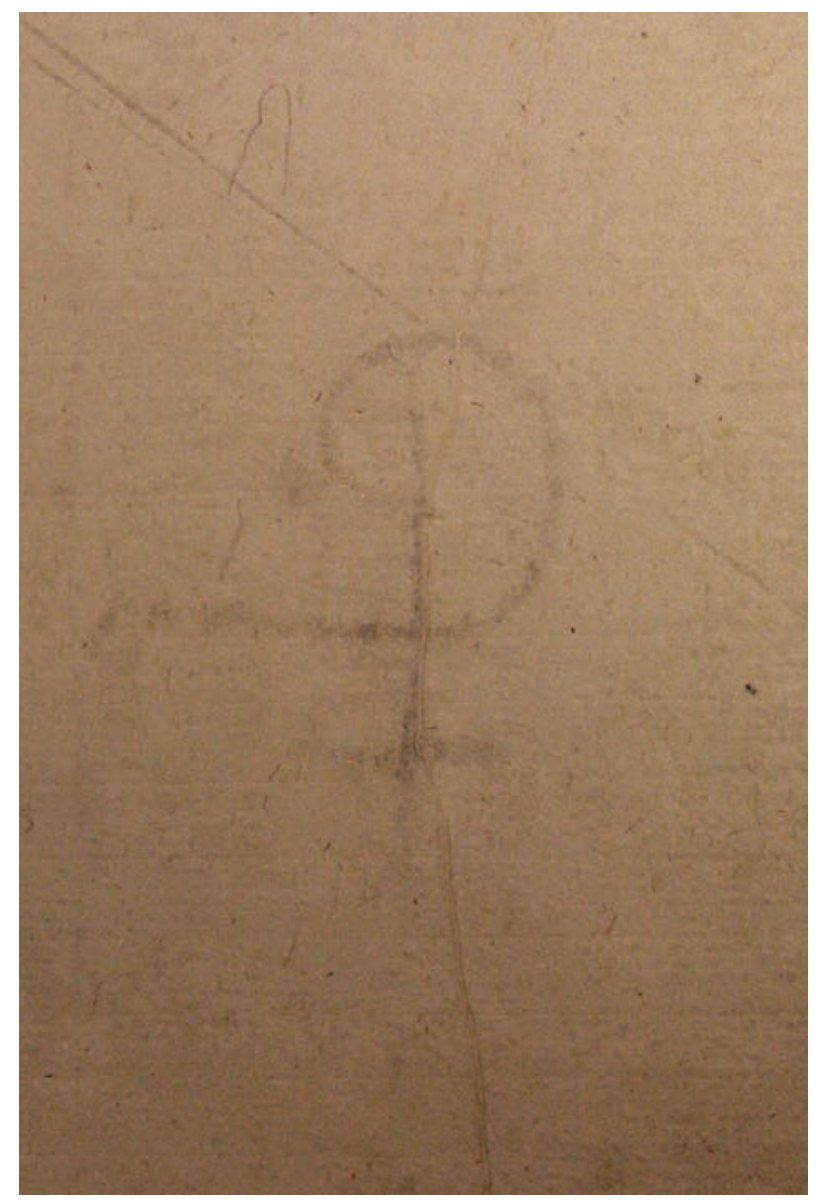

Fig. 1. Venezia, Biblioteca Nazionale Marciana, Marc. gr. Z. 516 (904), fol. 139r. Filigrana con lettera $G$

al $1367^{7}$, e inoltre una delle filigrane del testo della Geografia mostra forti analogie con due filigrane attestate a Genova fra 1318 e 1328, ambedue segnate nell'Archivio di Stato del capoluogo ligure come "Minute di Lanfranco de Nazario": una $\mathrm{G}$ sormontata da croce latina (fig. 1), che però è ruotata di novanta gradi rispetto agli esempi pubblicati nei repertori di Briquet e di Mošin e Traljić ${ }^{8}$. Bernardinello contesta questa proposta: "la $G$ non è ruotata di $90^{\circ}$ rispetto a filoni e vergelle, è di proporzioni notevolmente diverse da quelle dei facsimili riprodotti in Mošin e Briquet ed è sovrastata da croce latina, non da croce a doppia traversa", e propone un confronto con una filigrana inedita, riferita all'Archivio Datini 522 (Livorno, gennaio 1389) e conservata nell'Archivio di Stato di Prato, recuperata dall'archivio dei facsimile della collana Die Wasserzeichenkartei Piccard nell'Haupstaatarchiv di Stoccarda ${ }^{9}$. Al di là dell'impossibilità di constatare quanto scritto da Bernardinello (a distanza di quasi vent'anni dal suo articolo il materiale sulla lettera G della serie Die Wasserzeichenkartei Piccard giace ancora negli archivi di Stoccarda, inedito), ci sembra che la filigrana del Marc. gr. Z. 516 (904) possa essere una variabile intermedia fra i due esempi sopracitati e riferiti a Lanfranco di Nazario: l'esemplare del 1318 è infatti di dimensioni differenti, e nonostante la $\mathrm{G}$ sia ancora ruotata di $90^{\circ}$ rispetto all'esempio marciano, in questo caso è sormontata da una croce latina senza la seconda traversa. Ad ogni modo, per certificare la diffusione del motivo della $\mathrm{G}$ sormontata da una croce nel corso del Trecento, basti considerare un'altra coppia di filigrane, pubblicate da Mošin e Traljić ma non da Briquet, con una G sormontata da croce a doppia traversa dalla composizione analoga all'esempio marciano - fra l'altro orientata come quella - e a quelli genovesi, attestata a Mosca fra il 1355 e il $1370^{10}$. Le altre due filigrane nella Geografia raffigurano invece un cervo e un fiore stilizzato, quest'ultimo analogo a esempi utilizzati nel 1318 a Dubrovnik, sulla costa dalmata ${ }^{11}$. Questi primi dati codicologici permettono di individuare la stesura della Geografia e delle altre opere, di mano di Teluntàs, attorno al 1320, anticipando la proposta di Elpidio Mioni che invece si spingeva a proporre come terminus ante quem addirittura il $1340^{12}$.

\section{LE IMMAGINI DEI FASCICOLI AGGIUNTI.}

A corredo dei tre testi il Tolomeo marciano presenta, fuori numerazione, un binione iniziale (ff. 1-4) e due bifoli (ff. 140-141, 160-161) ${ }^{13}$, che illustrano teorie cosmologiche, diagrammi musicali, nel tentativo di mediare fra il sapere scientifico dell'antichità classica, oggetto di studi approfonditi e di una generale riscoperta durante l'età paleologa, e la teologia cristiana. Le fonti letterarie e filosofiche che guidano questa enciclopedia illustrata sono già state analizzate diffusamente da Mioni e da Furlan; di seguito si suggeriranno solo alcune integrazioni, per poi passare all'analisi stilistica delle immagini.

$\mathrm{Al}$ f. iv è raffigurato un argano che ruota incessantemente, in un movimento perfetto che regola la successione fra il giorno e la notte, le fasi lunari e quelle solari, il cielo e gli $\operatorname{astrir}^{14}$ (fig. 2). L'effetto artistico è straniante: mai il moto uni-

\footnotetext{
${ }_{7}^{7}$ D. JACOBI, The Latin Empire of Constantinople and the Frankish States in Greece, in The New Cambridge Medieval History, V: c. 1198-c. 1300, D. Abulafia (dir.), Cambridge 1999, p. 525-542.

${ }^{8}$ E. MIONI, Bibliothecae divi Marci Venetiarum codices Graeci manuscripti. Thesaurus antiquus. 2: Codices 300-625, Roma 1985, p. 381. Le filigrane sono pubblicate in: C.M. BRIQUET, Les filigranes. Dictionnaire historique des marques du papier dès leur apparition vers 1282 jusq'en 160o, Paris 1907, III, nn. 8184 e 8186, p. 443; V.A. MOŠIN e S.M. TRALJIĆ, Filigranes des XIII et XIV siècles, Zagreb 1957, nn. 5256 e 5258.

${ }^{9}$ S. BERNARDINELLO, op. cit (n. 4), p. 54, n. 23 e 24.

${ }^{10}$ V.A. MOŠIN e S.M. TRALJIĆ, op. cit. (n. 8), n. 5259 e 5260.

${ }^{\text {" }}$ Mioni non individua il cervo nei repertori, mentre per quanto riguarda il fiore lo lega al gruppo Mošin-Traljić n. 3974-3977, datati al 1318. E MIONI, op. cit. (n. 8), p. 381 .

${ }^{12}$ E. MIONI, op. cit. (n. 2), p. 58.

${ }^{13}$ BERNARDINELLO, op. cit., (n. 4), p. 51 n. 13

${ }^{14}$ Le illustrazioni dei ff. 1v-2r citano le teorie cosmologiche di Niceforo Cumno, ministro di Andronico II, che nelle sue opere tentò di mediare fra l'assunto aristotelico dell'eternità e la teologia cristiana che pretendeva una Creazione ex nihilo, teorizzando una duplice nozione di tempo: da un lato un tempo legato alla dimensione fisica dell'universo, scandito dal movimento dei pianeti, dall'altro invece un tempo divino e immutabilmente eterno, che invece

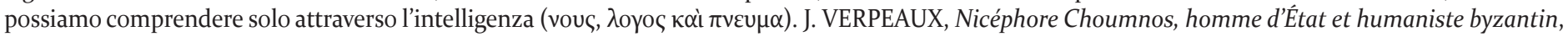
Paris 1959, p. 137; E. AMATO e I. RAMELLI, Filosofia rethoricans in Niceforo Cumno: l'inedito trattato Sui corpi primi e semplici, in Medioevo Greco, 6, 2006, p. 1-40; M. MARCHETTO, Nikephoros Chumnos'Treatise On Matter, in Aesthetics and Theurgy in Byzantium, (Byzantinisches Archiv. 25), S. Mariev e W.-M. Stock (dir.), Berlin 2013, p. 31-56.
} 


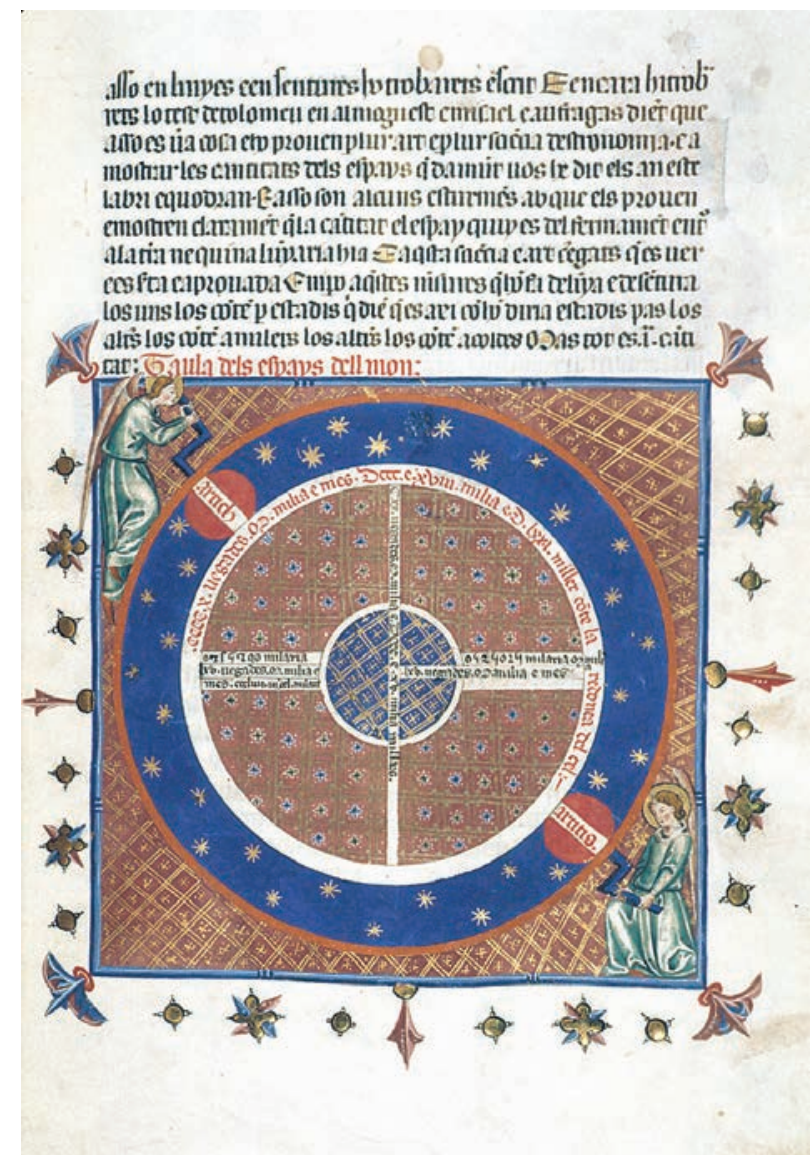

Fig. 3. Londra, British Library, Yates Thompson 31, fol. 45 r. Rappresentazione dell'universo.

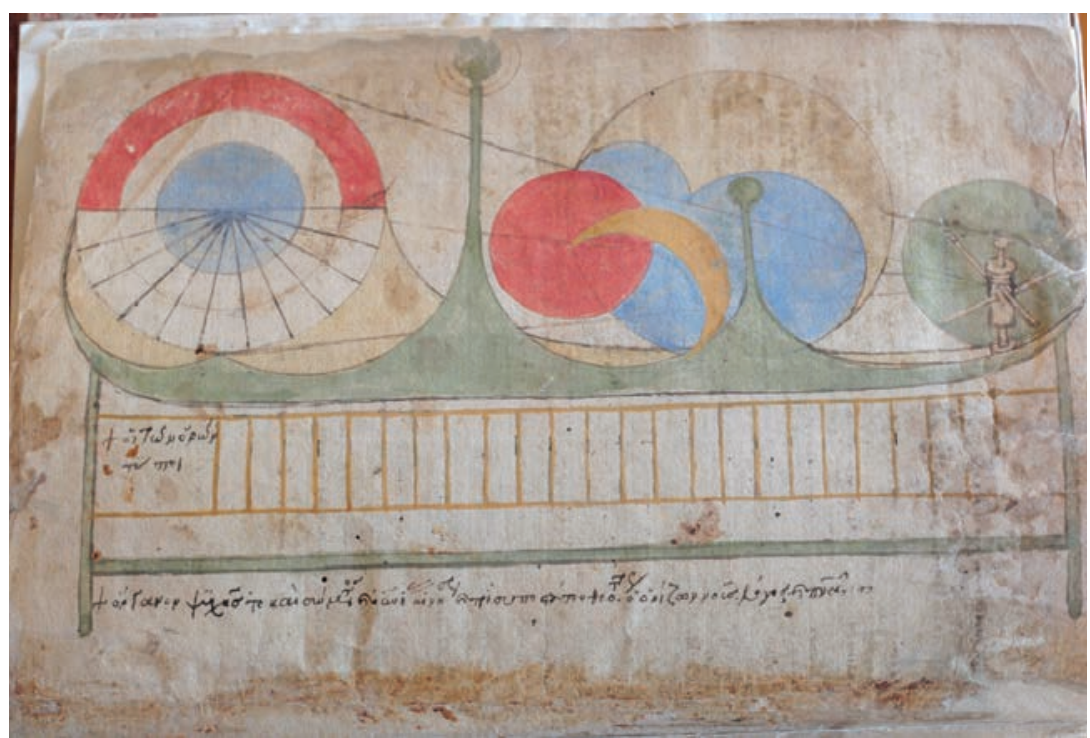

Fig. 2. Venezia, Biblioteca Nazionale Marciana, Marc. gr. Z. 516 (904), fol. 1v. Il Motore immobile dell'universo.

versale era stato rappresentato con tale chiarezza scientifica. Il ricorso all'argano, poi, svela insospettabili parentele con quanto avveniva negli stessi volgere d'anni dall'altro capo d'Europa, dove sempre ad un argano - ma questa volta mosso dagli angeli - ricorreva il miniatore del Breviari d'amor di Matfré Ermengau di Béziers per raffigurare il motore immobile l'universo (fig. 3$)^{15}$.

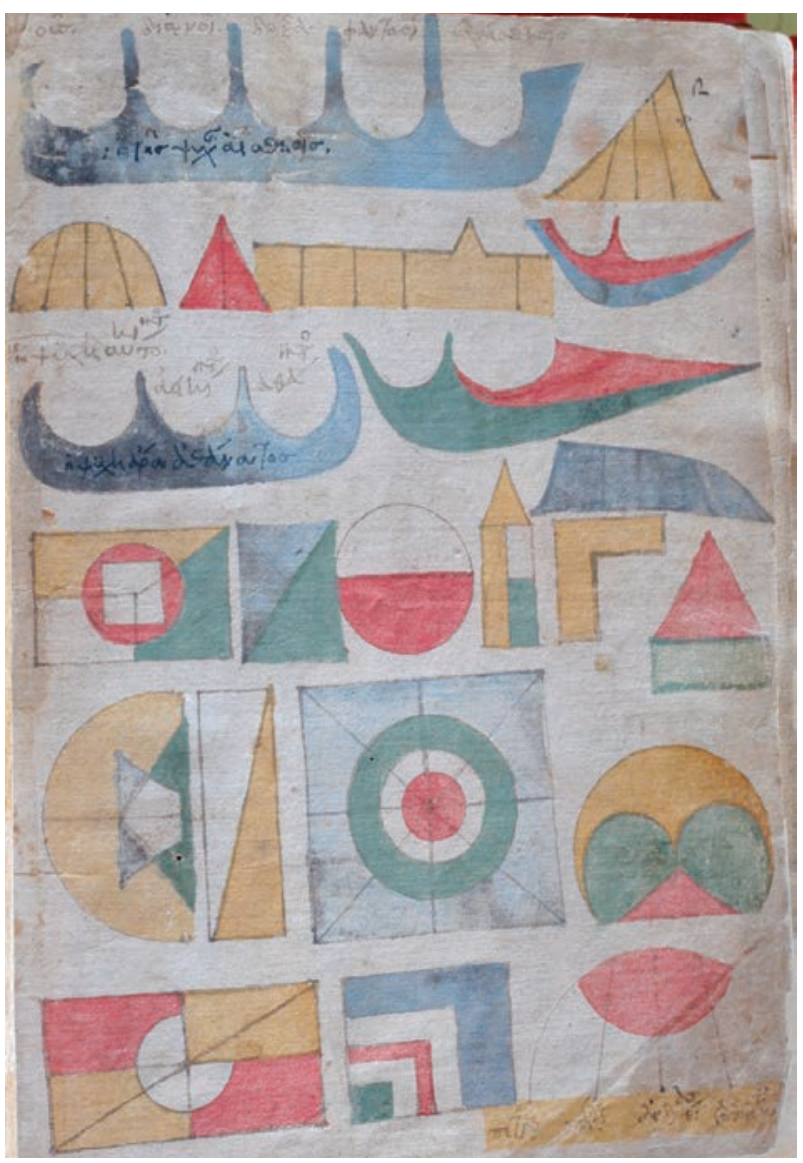

Fig. 4. Venezia, Biblioteca Nazionale Marciana, Marc. gr. Z. 516 (904), fol. 2r. Diagrammi sulla conoscenza

Al folio successivo alla chiarezza didascalica si sostituisce un'immagine ermetica, di ardua decifrazione (per noi): un'alternanza di figure geometriche assemblate fra loro (triangoli, cerchi e rettangoli che si uniscono, vivacemente colorati in rosso, verde, giallo, azzurro) e forme più libere, che a un primo sguardo sembrano quasi delle imbarcazioni avveniristiche (fig. 4). La figura in alto a sinistra evoca appunto una nave a quattro alberi, al cui interno si legge $(=\dot{\eta}) \tau \tilde{\eta} \varsigma \psi v \chi \tilde{\eta} \varsigma$ ai̋ $\sigma \theta \eta \sigma ı s$, mentre fra le anse del disegno sono elencate da destra a sinistra le tappe del percorso che l'uomo compie per affinare la propria conoscenza: dall'aí $\sigma \theta \varepsilon \sigma ı \zeta$, la percezione sensibile, il grado primigenio dell'apprendimento

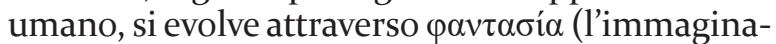
zione), la $\delta o ́ \xi \alpha$ (l'insieme delle opinioni, "non esenti da contraddizione" come scrisse nel V secolo Proclo

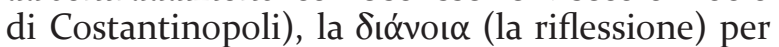
elevarsi infine al vous, la contemplazione pura, il superamento di tutte le idee. Ma quale è il significato delle singole figure? Secondo Aristotele l'uomo ha delle capacità innate di ordinare le proprie conoscenze, raggruppandole in classi e riuscendo a cogliere l'essenza propria di ciascuna di esse: nel libro VII e nel libro VIII della Metafisica lo Stagirita tratta ampiamente del concetto di sostanza e di materia, che nella sua opinione sono concetti inscindibili; anzi, la sostanza (ov̉oía) si nasconde all'interno della cosa sensibile come suo fondamento ontologico, e cioè

\footnotetext{
${ }^{15}$ Analogamente al Marc. gr. Z. 516 (904), anche il Breviari d'amor tenta di conciliare teologia e sapere enciclopedico, servendosi fra l'altro anche della cosmologia tolemaica. M. FRANKLIN-BROWN, The Lyric Encyclopedia: Citation and Innovation in Matfre Ermengaud's Breviari d'amor, in Romance Notes, 51/3, 2011, pp. 389-396. Sulla tradizione miniata del Breviari d'amor: C. MIRANDA GARCIA-TEJEDOR, Los manuscritos con pinturas del Breviari d'Amor de Matfre Ermengaud de Beziers. Un estado de la cuestion, in La miniatura medieval en la Península Ibérica, J. Yarza Luaces (dir.), Murcia 2007, p. 323.
} 


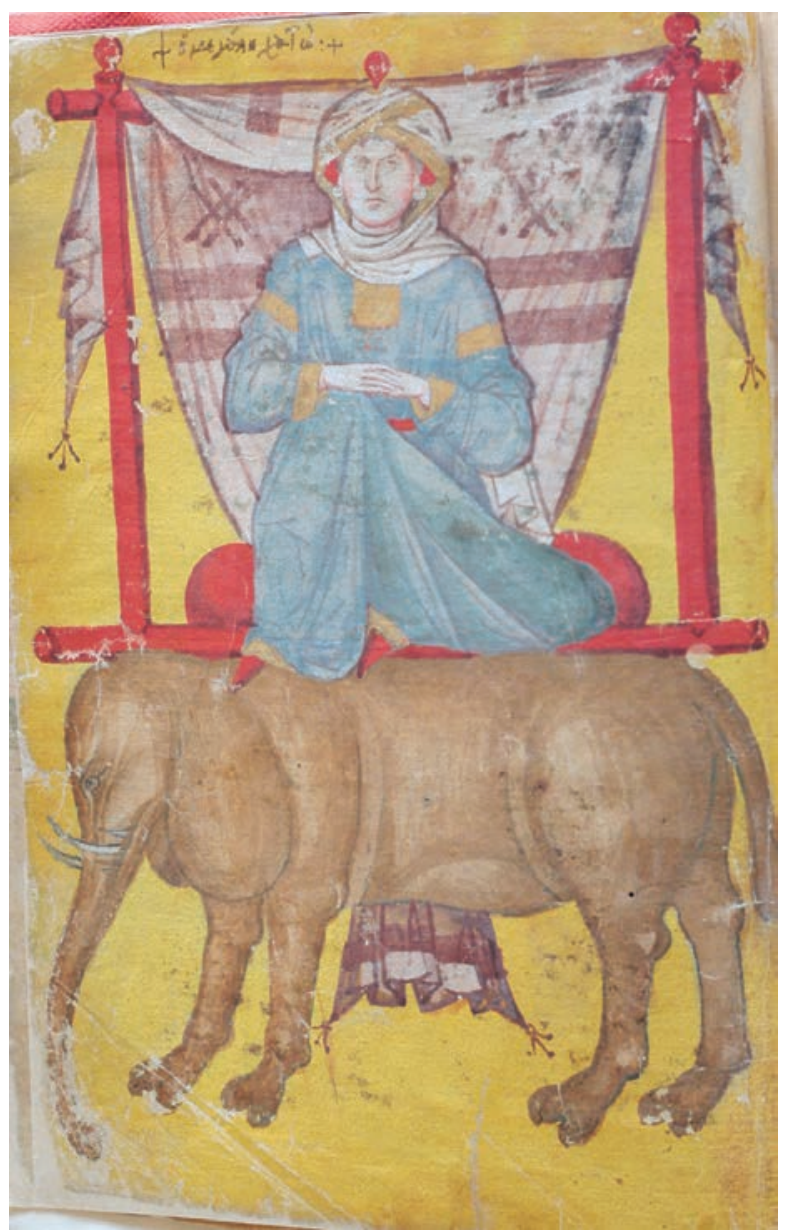

Fig. 5. Venezia, Biblioteca Nazionale Marciana, Marc. gr. Z. 516 (904), fol. 2v. Ritratto di gentildonna

la definisce come tale ${ }^{16}$. Da questo principio, l'interpretazione della miniatura al f. 2 r si muove lungo due direttrici complementari. Nel primo caso, si consideri il trattato Quod neque Materia ante Corpora ${ }^{17}$, che Niceforo Cumno dedicò alla nozione di materia: qui l'intellettuale bizantino confuta il concetto di "materia eterna" caro ad Aristotele, teorizzando invece che la sostanza e la sua forma sono il risultato della volontà di Dio, una creazione immediata e indissolubile che si deve alla Sua saggezza e volontà ${ }^{18}$. "Questo universo

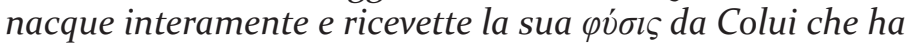
creato il mondo e l'ha ordinato"19 scrive Cumno, e ripete quanto scriveva san Basilio, secondo cui Dio mentre creava la forma concepì anche una materia in armonia con la forma ${ }^{20}$. In quest'ottica, nulla vieta di intendere la serie di forme e figure del f. 2 r come una rappresentazione di questa teoria, attingendo ad un immaginario asettico e "scientifico" che

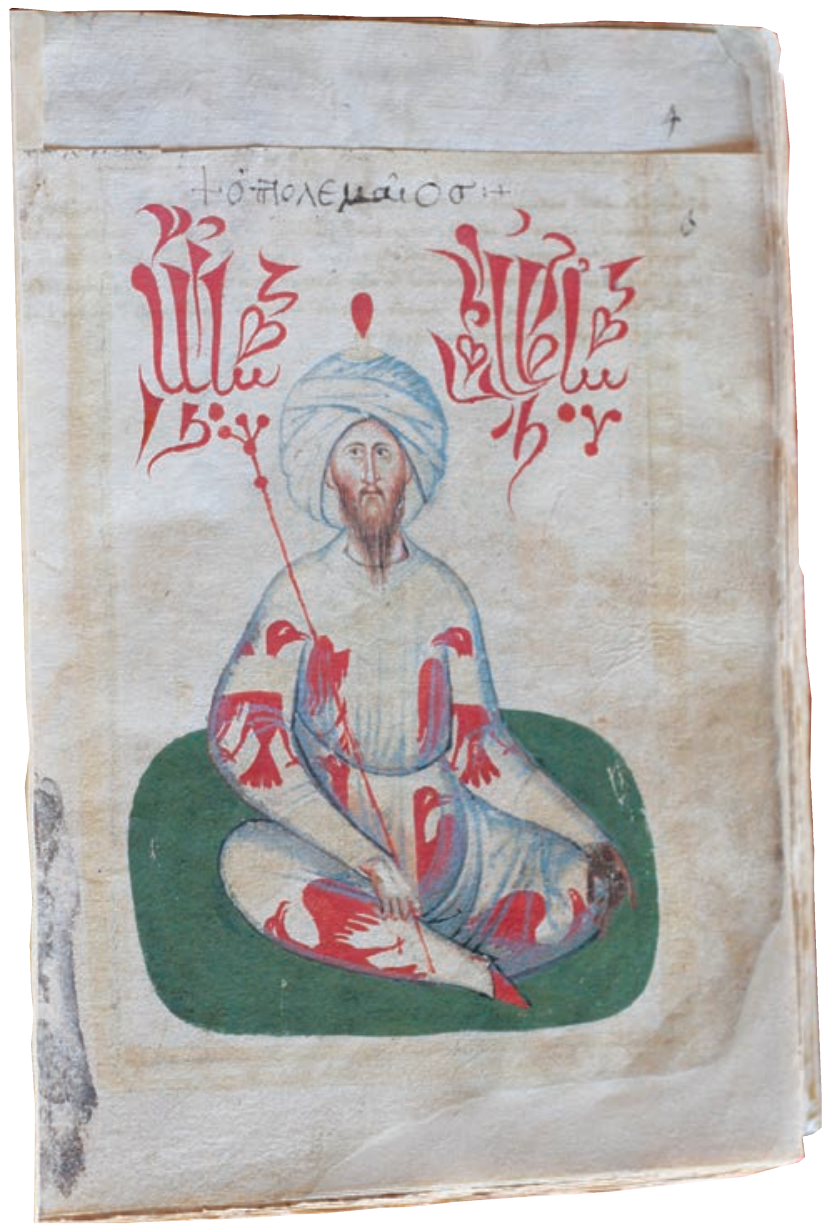

Fig. 6. Venezia, Biblioteca Nazionale Marciana, Marc. gr. Z. 516 (904), fol. 3r. Ritratto di Claudio Tolomeo

restituisca a un pubblico di iniziati le idee di Cumno sul concetto di forma e materia appena descritto.

Ma se decidessimo di spingere in un'altra direzione i nostri tentativi ermeneutici, dovremmo tornare agli scritti di Aristotele, e in particolare ai passi dedicati alla conoscenza. Nel De anima, il filosofo scrive: "la facoltà pensante pensa le sue forme in immagini mentali" ${ }^{21}$, e ancora "nessuno potrebbe mai imparare a capire qualcosa, se non avesse le facoltà della percezione; anche quando pensa speculativamente deve avere alcune immagini mentali con cui pensare" ${ }^{22}$; nel De memoria et reminiscentia rincara la dose: "Come è già stato detto nel mio trattato Sull'anima a proposito dell'immaginazione, è impossibile pensare senza un'immagine mentale" ${ }^{\prime 23}$. Il concetto di "immagine mentale" diventa centrale nello sviluppo della ars memorativa nel Medioevo occidentale, e il De memoria et reminiscentia, assieme al manuale di retorica Ad Herennium ${ }^{24}$

\footnotetext{
${ }^{16}$ ARISTOTLE, Metaphysics, VII, 1042a, H. Tredennick (dir.), Cambridge (MA) / London 1933, p. 400-403.

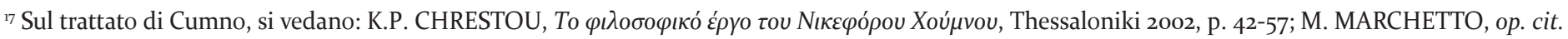
(n. 14).

${ }^{18}$ Si tratta di un'impostazione che Cumno recupera dalla patristica greca, in particolare da Gregorio di Nissa, il quale era giunto a conclusioni simili nella sua Apologia. M. MARCHETTO, op. cit. (n. 14), p. 46-47.

${ }^{19} \mathrm{Il}$ passo è tratto dalla traduzione in francese della lettera n. 42, edita in J. F. BOISSONADE, Anecdota Nova, Paris 1844, p. 52.

${ }^{20}$ P. DUHEM, Le système du monde. Histoire des doctrines cosmologiques de Platon à Copernic. 2. La cosmologie hellenique (suite); L'astronomie latine au Moyene Age, Paris 1954, p. 428.

${ }^{21}$ ARISTOTLE, De anima, III, 431b, 2 in ARISTOTLE, On the Soul. Parva Naturalia. On Breath, W. S. Hett (ed.), Cambridge (MA) / London 1936, p. 176.

${ }^{22}$ Ibidem, 432a, 9, p. 180.

${ }^{23}$ ARISTOTELE, De memoria et reminescentia, 449b, 31 in ARISTOTLE, On the Soul op. cit. (n. 21), p. 290.

${ }^{24}$ Per tutto il Medioevo, e fino al Rinascimento, la Rhetorica ad Herennium fu considerato un'opera di Cicerone: P.F. GRENDLER, Schooling in Renaissance Italy: Literacy and Learning, 1300-1600, Baltimore 1989, p. 115-116 e 212-214; V. COX, Macchiavelli and the Rhetorica ad Herennium: Deliberative Rhetoric in The Prince, in The Sixteenth Century Journal, 28, 1997, p. 1114-1117.
} 
e al De oratore di Cicerone ${ }^{25}$, sarà il testo capitale su cui fonderanno le loro teorie mnemotecniche sia Alberto Magno che Tommaso d'Aquino ${ }^{26}$. Purtroppo fra le opere di Cumno non ne sopravvive alcuna che si dedica al concetto di memoria, né Verpaux ipotizza un qualche interesse da parte dello statista bizantina su questi argomenti; istituire dunque una relazione diretta fra l'immagine nel Marc. gr. Z. 516 (904) e gli studi sull'arte della memoria che qualche decennio prima occuparono i filosofi scolastici risulta dunque poco proficuo, ma non è da escludere completamente - proprio per la sua indecifrabilità, e per il carattere innovativo della composizione - che l'artista abbia qui voluto dipingere un'embrionale ars memorativa bizantina, affidando a ciascuna figura il ruolo di imago agentes per raggiungere il vouc, quasi che il foglio - contraltare dell'esattezza geografica delle tavole di Agatodemone di Alessandria - contenesse le coordinate verso la conoscenza assoluta, el'imbarcazione a quattro alberi fosse "una navicella dell'anima nel suo tragitto verso gli intellegibili"27.

Questo primo assaggio filosofico si interrompe appena voltata la pagina, di fronte a due ritratti a pieno foglio di pregevole fattura: una donna e un uomo, entrambi indossano un turbante con un diadema e le scarpette purpuree, simboli regali (figg. 5-6). Il ritratto femminile (f. $2 \mathrm{v}$ ) spicca per l'ingombrante presenza di un elefante - la cui mole è comunque ridotta rispetto alle dimensioni della donna - quasi sul punto di cedere allo sforzo, mentre sopra di lui la donna siede entro un baldacchino appoggiando le mani al ginocchio destro. Lo sguardo altèro e la smorfia delle labbra stonano con l'eleganza augustea che suggerisce la gran profusione di dettagli purpurei, dalla struttura del baldacchino alle scarpette fino alla più minuziosa fodera interna del turbante o alla cintura intorno alla vita, restituendoci un'immagine ambigua, ma viva.
Al f. $3 r$ l'uomo invece risponde ai canoni estetici aristocratici: seduto alla turca, tiene nella destra un lungo scettro e indossa un caftano bianco ricamato col simbolo dell'aquila a doppia testa, che all'inizio del XIV secolo entrò stabilmente nell'iconografia imperiale bizantina fino ad essere adottato ufficialmente dai Paleologhi nel $1325^{28}$. Il ritratto è accompagnato da due svolazzanti iscrizioni in inchiostro rosso, circondate da orpelli e arabeschi che tentano di mascherare l'imitazione maldestra della grafia araba della parola Sultàn, copiata malamente su entrambi i lati da uno scriba digiuno di arabo ${ }^{29}$. Entrambi i ritratti sono accompagnati inoltre da un'iscrizione greca ciascuno: sul margine superiore del f. $2 \mathrm{~V}$ Mioni legge $\dot{\eta} \mu \varepsilon \gamma \alpha \dot{\alpha} \eta \eta \chi \alpha ́ \tau \omega$, mentre sopra il ritratto maschile è stato aggiunto in inchiostro nero ó $\pi \tau 0 \lambda \varepsilon \mu \alpha \alpha_{0}{ }^{30}{ }^{30}$. La didascalia $\dot{\eta} \mu \varepsilon \gamma \alpha \dot{\alpha} \eta \eta \chi \alpha ́ \tau \omega$ è stata tradizionalmente sciolta come "la grande gentildonna", muovendo dall'interpretazione di $\chi \alpha ́ \tau \omega$ quale grecismo del termine arabo chatun, gentildonna appunto: questo singolo dettaglio ha tratto in inganno Olshausen, che vi lesse la traduzione in greco di Sitt-Chatun (la grande gentildonna), andata in sposa a Maometto II nell'inverno 1449 prima di essere abbandonata pochi mesi dopo ad Adrianopoli mentre il marito proseguiva la vittoriosa campagna verso Costantinopoli ${ }^{1}$. Su queste basi, il ritratto accanto non poteva che essere strettamente correlato con la Sitt-Chatum, e su queste direzioni muovono le interpretazioni dell'iscrizione pseudo-araba: Giovanni Morelli, in una manoscritto conservato alla Biblioteca Marciana, cita alcuni esperti da lui interpellati che vi lessero in entrambi i lati il nome Selim², Olshausen suppone vi sia scritto Sulla Slla, da cui ricostruisce Sultan Aslan, signore di Elbistan in Anatolia (1454-1466) e soprattutto fratello della Sitt-Chatun ${ }^{33}$, mentre Babinger lo pubblica semplicemente come ritratto di Maometto I[ ${ }^{34}$.

\footnotetext{
${ }^{25} \mathrm{Nel}$ De oratore Cicerone racconta la storia del poeta Simonide e della strage in casa del patrizio Scopa, l'evento a cui si fa risalire l'invenzione dell'arte della memoria: CICERO, De oratore, II, LXXXVI, E. W. Sutton e H. Rackham (dir.), Cambridge (MA) / London 1959, p. 352-353.

${ }^{26}$ Entrambi fra l'altro scriveranno un commentario al De memoria et reminiscentia di Aristotele, muovendo dalla traduzione che delle opere aristoteliche fece Guglielmo di Moeberke. Appartenente all'ordine dei domenicani, Guglielmo fu eletto arcivescovo di Corinto nel 1278, e rimase in carica - nonostante si abbia prova di ripetuti soggiorni in Italia e di missioni in Europa per conto della corte papale - fino alla morte, avvenuta nel 1286. È possibile ipotizzare che Guglielmo intrattenne qualche rapporto con gli intellettuali greci suoi contemporanei, ma gran parte della sua attività di traduttore si svolse prima della nomina ad arcivescovo, e allo stato attuale delle ricerche non è possibile documentare se il suo lavoro influenzò in qualche modo la riscoperta dell'opera di Aristotele anche a Bisanzio. Una curiosità: Tommaso d'Aquino tramanda che Guglielmo tradusse anche un'opera (oggi perduta) il cui titolo in latino era De aquarum conductibus et ingeniis erigendis, che si ipotizza fossero i Pneumatica di Erone di Alessandria, contenuti nel Marc. gr. Z. 516 (904). Per approfondire la figura di Guglielmo di Moeberke, e la sua attività di traduttore, si vedano: Guillaume de Moerbeke. Recueil d'études à l'occasion du 700 anniversaire de sa mort, 1286, J. Brams e W. Vanhamel (dir.), Leuven 1989; J. BRAMS, La riscoperta di Aristotele in Occidente, Milano 2003, p. 105-130; E. FRYDE, The Early Paleaeologan Renaissance (1261-C.1360), Leiden / Boston / Köln 200o, p. 103-143. Sulle arti della memoria nel Medioevo, si vedano: F.A. YATES, The Art of Memory, London 1966; M. CARRUTHERS, The Book of Memory: A Study of Memory in Medieval Culture, Cambridge 1992.

${ }^{27}$ I. FURLAN, Il libro op. cit. (n. 2), p. 115.

${ }^{28}$ R. OUSTERHOUT, The Byzantine Heart, in Zograf, 17, 1986, p. 36-44. Un altro simbolo legato alla dinastia paleologa è l'asterisco \# che decora le tende del baldacchino, alle spalle della nobildonna al f. 2v: B. HEMMERDINGER, Deux notes d'héraldique, in Byzantinische Zeitschrift, 61/2, 1968, p. 304-309.

${ }^{29}$ Devo a Giuseppe Mandalà, che qui ringrazio, la lettura delle due iscrizioni.

$3^{\circ}$ E. MIONI, op. cit. (n. 8), p. 381.

${ }^{31}$ La vicenda è raccontata dallo storico bizantino Michele Duca: DUKAS, Istoria Turco-Bizantina (1341-1462), V. Grecu (ed.), Bucaresti 1958, p. 279-281. Sull'identificazione dell'immagine con la Sitt-Chatun, si veda: J. OLSHAUSEN, Eine Merkwürdige Handschrift der Geographie des Ptolemeus, in Hermes, 15, 188o, p. 417-424. L'ipotesi è accolta da Babinger, che riprodusse l'immagine come ritratto della stessa: F. BABINGER, Mehmeds II Heirat mit Sitt-Chatun (1449), in Der Islam, 29, 1949, p. 230-234; F. BABINGER, Maometto il Conquistatore e il suo tempo, Torino 1957, p. 258.

${ }^{32}$ Venezia, Biblioteca Nazionale Marciana, Cod. Riservato 94, p. 281. Il nome potrebbe riferirsi tanto al re biblico Salomone, di cui Selim è la derivazione araba, oppure a uno dei sultani ottomani che governarono Costantinopoli nella prima metà del Cinquecento: Morelli non ne specifica l'identificazione.

33 J. OLSHAUSEN, op. cit. (n. 31), p. 417-424. I soggetti sono identificati come Sitt-Chatun e Melik Arslan ancora nel catalogo Byzantium. Faith and Power (1261-1557): qui Redford ipotizza che la donna sia ritratta mentre officia al suo stesso matrimonio, celebrato secondo l'eccentrico rito della prokypsis, assenti le autorità religiose e lo stesso sposo: lo studioso cita qui il precedente delle nozze fra Teodora, la figlia di Giovanni IV Cantacuzeno, e il sultano Orhan, che si celebrarono a Selymbria nel 1346 alla presenza della sola sposa e della famiglia di lei. Dunque, quella raffigurata nel codice marciano sarebbe una sorta di prokypsis "mobile", che la Sitt-Chatun avrebbe celebrato sul dorso dell'elefante, animale regale per eccellenza nel mondo islamico orientale (e, con un po' di ironia, questo potrebbe spiegare anche l'espressione stizzita della sposa). Secondo Redford, il doppio ritratto potrebbe essere stato eseguito quale dono per Melik Arslan, che conosceva il greco, proprio in occasione delle nozze della sorella. S. REDFORD, Byzantium and the Islamic World, 1261-1557, in Byzantium. Faith and Power (1261-1557), catalogue of the exhibition (New York, The Metropolitan Museum of Art, 23 marzo - 4 luglio 2004), H.C. Evans (dir.), New York 2004, p. 395.

${ }^{34}$ F. BABINGER, Mehmeds II op. cit. (n. 31), pp. 230-234; F. BABINGER, Maometto op. cit. (n. 31), p. 259.
} 


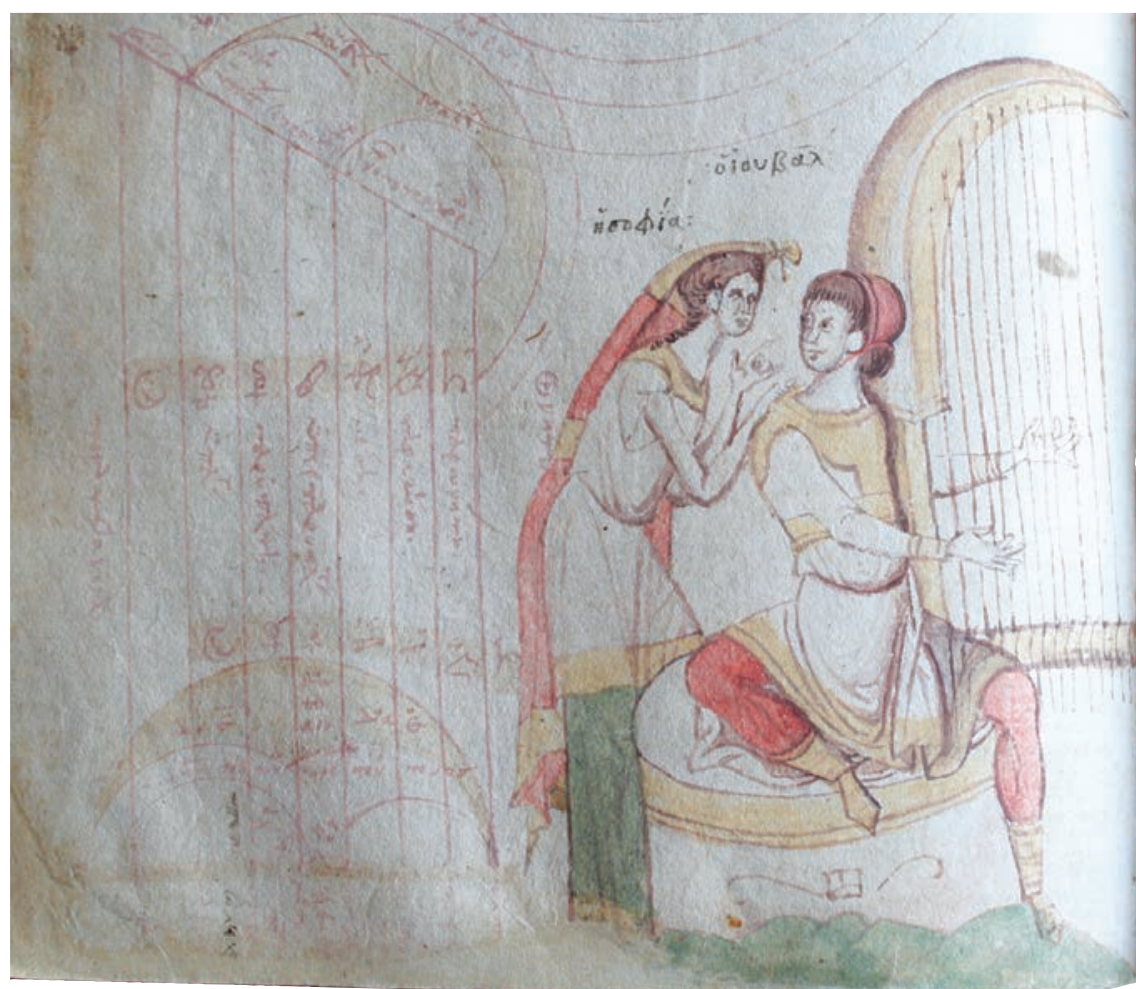

Fig. 7. Venezia, Biblioteca Nazionale Marciana, Marc. gr. Z. 516 (904), fol. 140v. Jubal e la personificazione della Saggezza.

Restano misteriosi i motivi per cui l'intera struttura ermeneutica dovrebbe poggiarsi esclusivamente su due iscrizioni, tacendo invece della terza, ó $\pi \tau 0 \lambda \varepsilon \mu \alpha i ̃ o s$, tanto più che entrambe le didascalie in lingua greca sembrano delle aggiunte posteriori alla realizzazione delle miniature ${ }^{35}$. Non è da escludere dunque che quanto noi oggi leggiamo sia il tentativo di riconoscimento operato da qualcuno nei decenni successivi alla realizzazione del manoscritto: ma se da un lato l'associazione fra il sultano e Claudio Tolomeo sembra giustificata, considerata l'omonimia dello scienziato alessandrino con i Tolomei, la dinastia che governò l'Egitto in età ellenistica, tanto che nel Medioevo si pensava che lo stesso Claudio fosse stato un sovrano, dall'altro $\dot{\eta} \mu \varepsilon \gamma \alpha \dot{\alpha} \lambda \eta$ $\chi \alpha ́ \tau \omega$ sembra un giochetto enigmistico, destinato più a intorbidire le acque che a schiarirle. La grande gentildonna potrebbe allora essere una personificazione, magari della Geografia - ma resterebbe inspiegabile lo sguardo torvo, ora che finalmente Massime Planude le ha ridato gloria dopo secoli di oblio - oppure del continente africano, esaltato dai dettagli esotici dell'abbigliamento e dell'elefante, ma contrariato da secoli di dominazione musulmana, oppure più banalmente un indefinito ritratto della moglie del "sultano" Tolomeo. Forzature, si dirà giustamente: fedele al motto agostiniano remota longissime ab intellectu profanorum ${ }^{36}$, l'immagine si cela ai nostri occhi, e rimane accessibile solo alla "compagnia degli amici" in grado di leggerne il significato.

In coda alle carte geografiche vennero inserite le "miniature musicali", che descrivono il rapporto fra l'armonia musicale e il movimento delle sfere celesti. In età paleologa, lo studio della musica faceva parte del quadrivium (aritmetica, musica, geometria, astronomia), dopo che Giovanni Pachimere $(\dagger 1310)$ aveva compilato il suo $\Sigma v ́ v \tau \alpha \gamma \mu \alpha$, elencando i punti cardine dell'educazione bizantina secondo l'antica suddivisione operata nel $\mathrm{V}$ secolo da Marziano Cappella ${ }^{37}$. La lunga iscrizione al $\mathrm{f}$. 140r introduce alla questione, elencando i protagonisti degli studi astronomici e musicali ${ }^{3}$ : da principio si ricorda Seth, figlio di Adamo, che assieme ai fratelli si dedicò allo studio degli astri e incise le sue scoperte su due colonne, nel timore che l'umanità potesse scordarle ${ }^{39}$; è poi il turno di Jubal, discendente di Caino e figlio di Lamech, che nella Genesi è descritto come "il padre di tutti coloro che suonano l'arpa e l'organo"40 e inventore - secondo Flavio Giuseppe - dello psalterion e della kithara ${ }^{41}$, mentre l'ultimo posto spetta a Pitagora, che aveva appreso l'astronomia e l'armonia musicale dai dotti al servizio del faraone, a loro volta istruiti da $\mathrm{Abramo}^{42}$. Accanto all'iscrizione appare il disegno di un tetracordo, che secondo Furlan ricalca da vicino le parole di un trattato sull'armonia musicale scritto alla fine del Duecento, gli Harmonica di Manuele Briennio ${ }^{43}$.

Il programma prosegue al folio successivo, dove l'artista ritrae Jubal, impegnato a suonare la lira, e la personificazione della Sophia in abiti classicheggianti (fig. 7); attorno a loro, la volta celeste, con al centro la Terra - indicata dall'iscri-

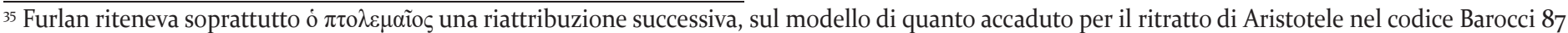
della Bodleian Library di Oxford, ribattezzato nel XV secolo come Argyropoulos: I. FURLAN, Codici greci op. cit. (n. 2), p. 39. Di diverso avviso Elpidio Mioni, che ravvisava una tale somiglianza fra l'iscrizione al f. zr e il resto del manoscritto da ritenerla della stessa mano del copista Andrea Teluntàs: E. MIONI, op. cit. (n. 2), p. 6o.

${ }^{36}$ SANCTI AURELII AUGUSTINI, Contra Academicos, I, I, 1, W.M. Green (dir.), Turnholti 1970, p. 3.

${ }_{37}$ Sugli studi scientifici durante l'epoca paleologa si veda: E. FRYDE, op. cit. (n. 26), p. 337-356 (con bibliografia precedente).

$3^{8}$ E. MIONI, op. cit. (n. 2), p. 62.

${ }^{39}$ Secondo la leggenda, di origine ebraica, Adamo predisse la distruzione la terra; Seth e i suoi fratelli decisero dunque di erigere due colonne, una in mattoni e l'altra in marmo, dove incisero le loro scoperte astronomiche con la speranza che queste sopravvivessero alla catastrofe. Alla fine, mentre quella di mattoni fu spazzata via dal Diluvio Universale, la colonna di marmo resistette, e - scrive Flavio Giuseppe nelle Antichità giudaiche - "esiste ancora oggi nel paese di Seirah". JOSEPHUS, Jewish Antiquities. Books I-IV, I, 68-71, H.S.J. Thackeray (dir.), Cambridge (MA) / London 1930, p. 32.

${ }^{40}$ Genesi, IV, 20-21.

${ }^{41}$ JOSEPHUS, op. cit. (n. 39), I, 64, p. 28-30.

${ }^{42}$ Pitagora inoltre ripristinò la lira, uno strumento inventato in Egitto da Tepandro e trasmesso a Orfeo, che a sua volta lo aveva consegnato a Pitagora. C.L. JOOST-GAUGIER, Pitagora e il suo influsso sul pensiero e sull'arte, Roma 2008, p. 153.

${ }^{43}$ Il canone musicale teorizzato da Briennio era basato sull'uso del numero, con l'obiettivo di realizzare l'armonia perfetta: questa ricerca lo portò appunto a riscoprire i testi antichi dedicati alla teoria musicali, fra i quali gli Harmonica di Claudio Tolomeo e gli scritti di Aristosseno. G.H. JONKER, The Harmonics of Manuel Bryennium, Groningen 1970, p. 57-59; I. FURLAN, Jubal e Pitagora in "miniature musicali" della Marciana, in Sine musica nulla disciplina... Studi in onore di Giulio Cattin, F. BERNABEI e A. LOVATO (a cura di), Padova 2006, p. 367. 


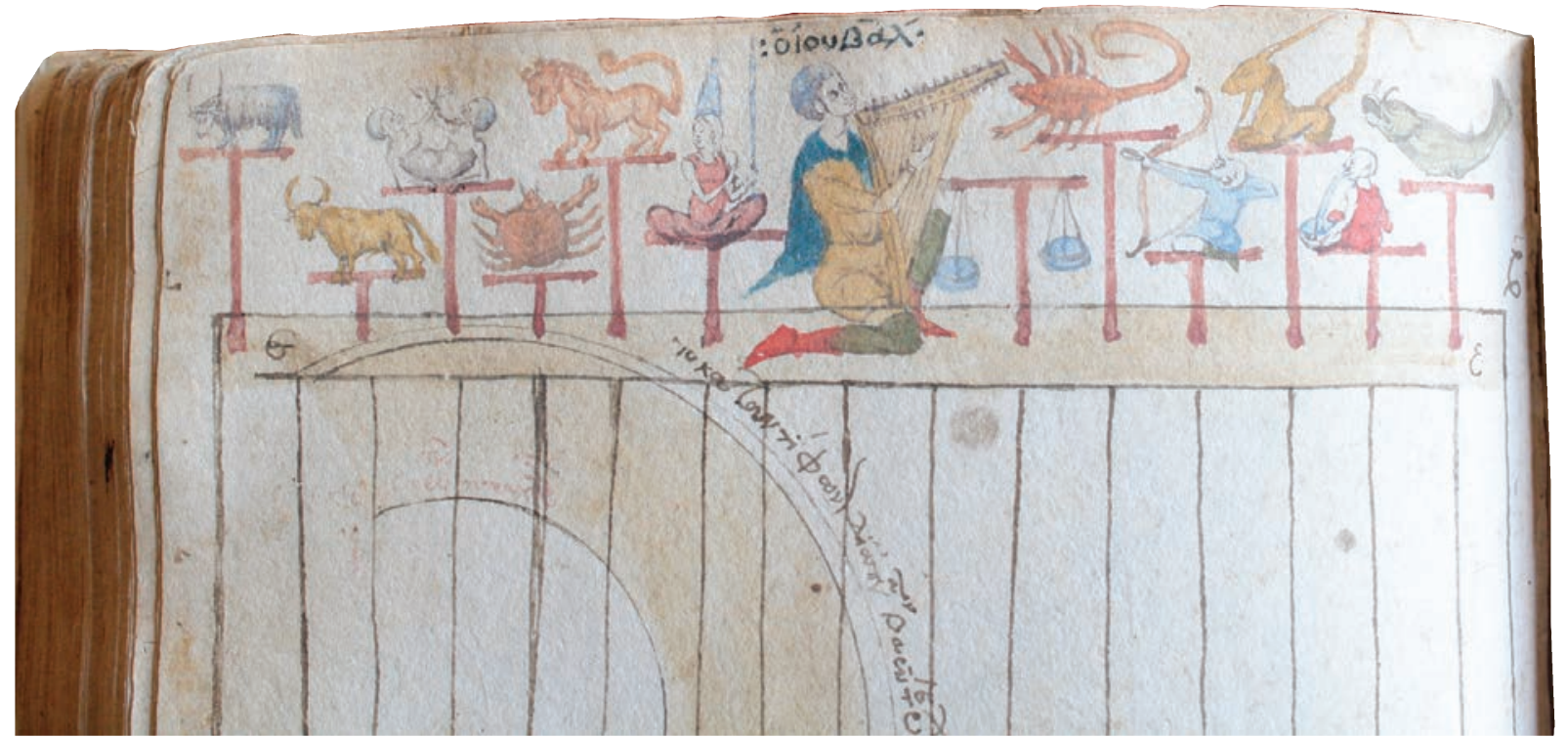

Fig. 9. Venezia, Biblioteca Nazionale Marciana, Marc. gr. Z. 516 (904), fol. 141v. Jubal e i simboli dello zodiaco

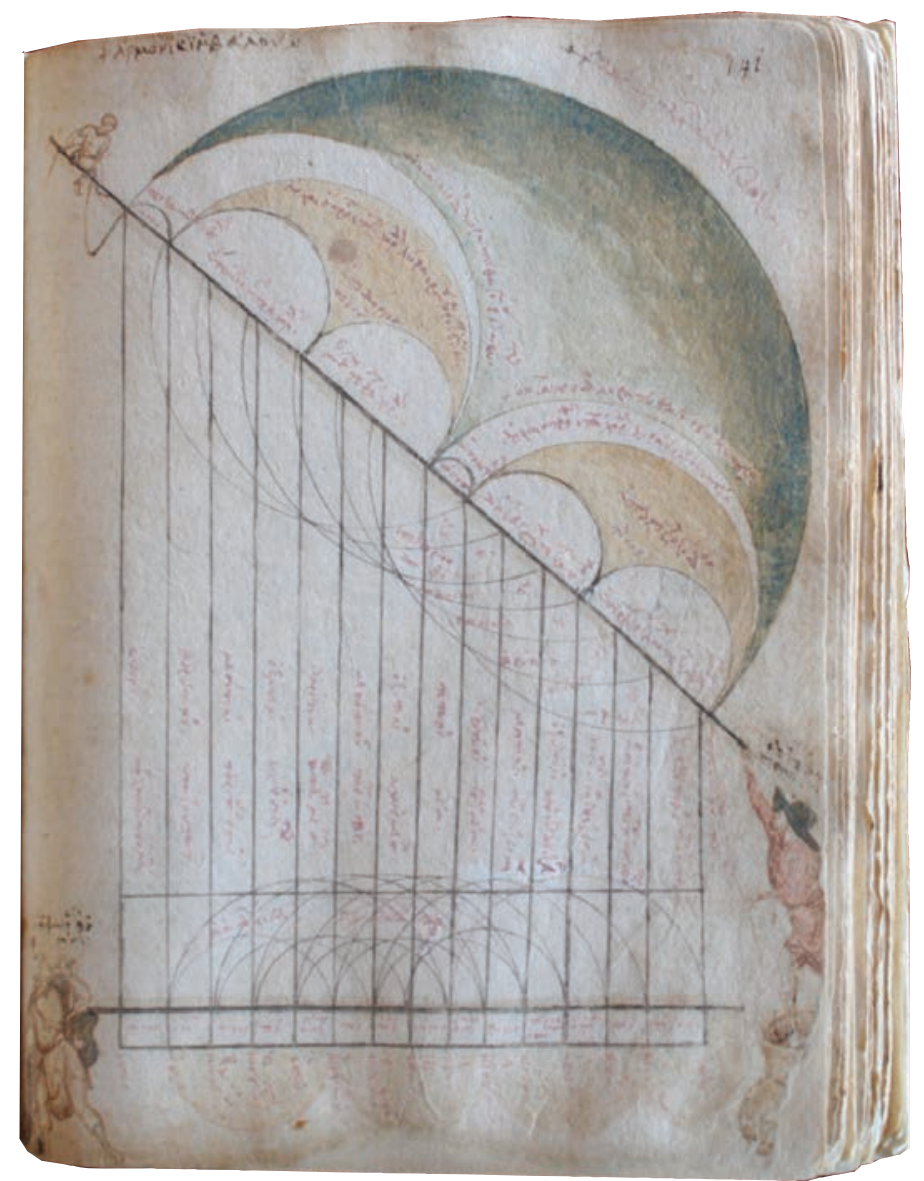

Fig. 8. Venezia, Biblioteca Nazionale Marciana, Marc. gr. Z. 516 (904), fol. 141r. Pitagora e il diagramma del sistema tonale della doppia ottava

zione $\Gamma \mathrm{H}$ - e attorno le orbite di sette pianeti, con le note dell'eptacordo che corrispondo a ciascuno di essi ${ }^{44}$. Lo sche- ma dell'eptacordo si interseca con un secondo diagramma, riferito questa volta a Pitagora e all'introduzione dell'ottava corda nella lira per ottenere la consonanza d'ottava, risolvendo quindi la dissonanza fra la corda più alta e la corda più bassa tipica della lira a sette corde. La didascalia posta in alto a sinistra riferisce proprio di Pitagora e del suo sistema a otto corde; le altre due che seguono invece ricordano ancora Jubal e Palamede, eroe dell'epopea troiana: il primo come semplice inventore di strumenti musicali, mentre al secondo

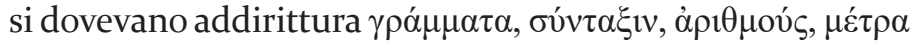

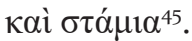

La miniatura al f. 141r raffigura a tutta pagina il sistema tonale della doppia ottava (fig. 8), ancora una volta un'invenzione di Pitagora; anzi, del sapientissimo Pitagora (ó

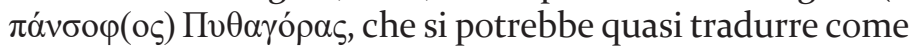
"il tuttologo Pitagora", segno della grande ammirazione che l'autore del Marc. gr. Z. 516 (904), come pure Manuele Briennio, avevano nei confronti del filosofo e matematico greco), come è indicato dall'iscrizione accanto al ritratto che lo vede raffigurato in equilibrio instabile, sopra una giara, mentre sembra cercare l'equilibrio appoggiandosi proprio allo schema armonico. Sul lato opposto, una fanciulla avvicina la mano al volto nell'atto di urlare: è la personificazione del suono e della voce, come da iscrizione

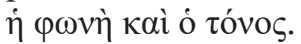

L'ultimo diagramma(f. 141v) si riferisce alla divisione del canone armonico secondo Briennio ${ }^{46}$, e anche in questo caso l'artista non si limita a riportare il nudo schema, ma una volta tracciato con riga e compasso il grafico, lo impreziosisce con miniature che ne sottolineano la grande familiarità con i temi trattati: qui, sopra la rappresentazione del canone, ricompare al centro Jubal (o ’Iovßód $\lambda$ impegnato a suonare la lira, con ai lati i dodici simboli dello zodiaco (fig. 9).

\footnotetext{
${ }^{44}$ Secondo Briennio, l'armonia perfetta e la melodia virtuosa che lo schema eptacordo genera possono essere intese solo da coloro che hanno purificato il loro spirito e il loro udito, coloro che - in accordo con lo schema descritto al f. $2 \mathrm{r}$ - hanno raggiunto il vouc. I. FURLAN, op. cit. (n. 43), p. 369.

${ }^{45}$ La fama di Palamede quale inventore era proverbiale nell'Antichità; in un frammento del sofista Alcidamante, risalente al IV secolo a.C., si legge che Palamede era l'inventore della musica. ALCIDAMANTE, Odisseo, 22, in Alcidamante. Orazioni e frammenti, G. Avezzù (ed.), Roma 1982. L'accenno a Palamede potrebbe inoltre nascondere un omaggio di Teluntàs alla sua città natale: Nauplia era infatti stata fondata dall'eroe eponimo Nauplio, padre di Palamede, mentre il colle che sovrasta la città costiera si chiama Palamidi.
}

${ }^{46}$ G.H. JONKER, op. cit. (n. 43), p. 53. 


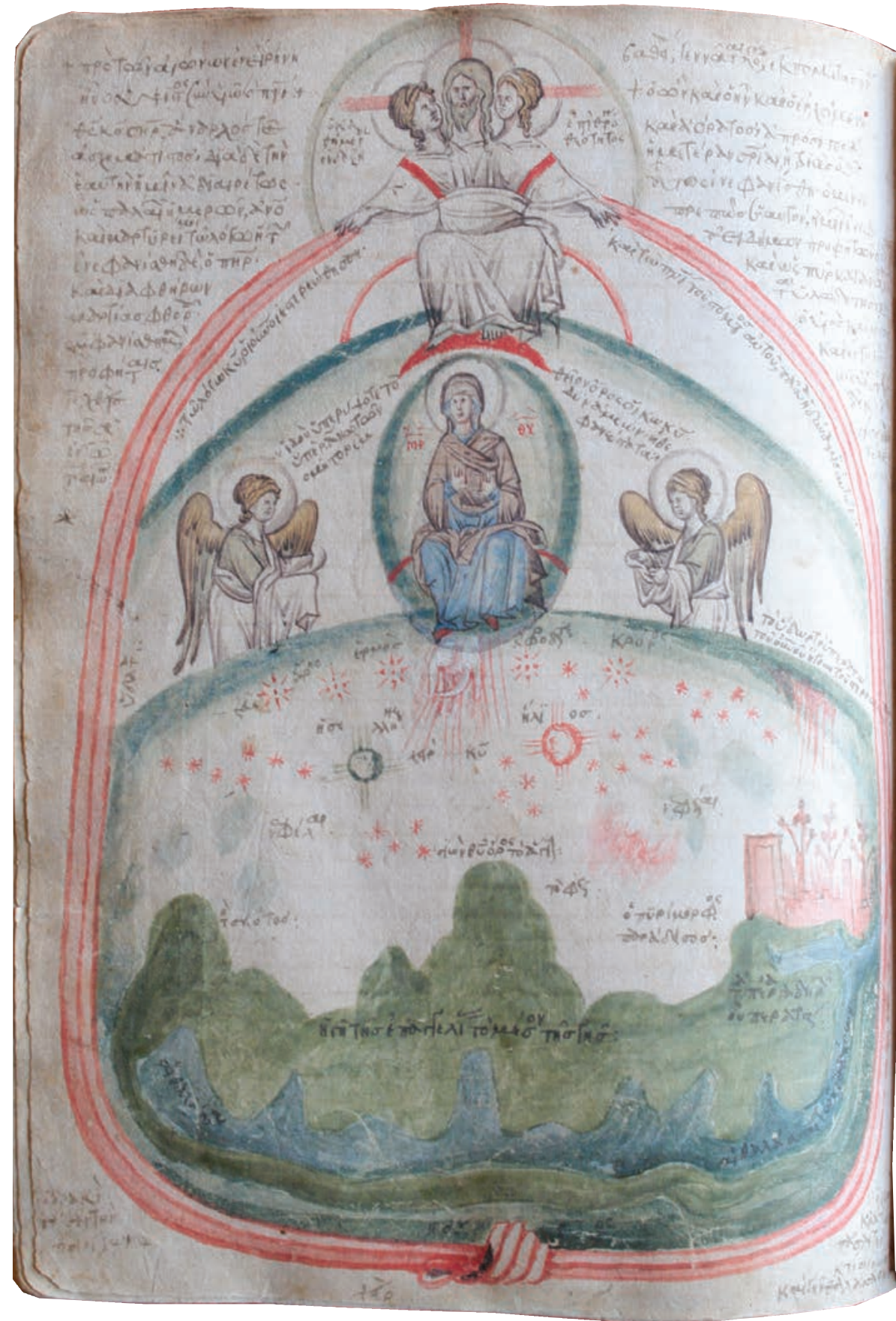

Fig. 10. Venezia, Biblioteca Nazionale Marciana, Marc. gr. Z. 516 (904), fol. $158 v$. Orbis christianus.

Nell'ultima sezione si compie l'associazione finale fra le tematiche cosmologiche e quelle religiose, attraverso quattro tavole dal forte valore simbolico, tutte accompagnate da lunghe didascalie che ne svelano il significato. La navicella ha finalmente raggiunto il voṽ, , e si appresta a esplorarlo.

Al f. 158v è dipinto l'orbis christianus (fig. 10), suddiviso in tre differenti parti e dominato dalla raffigurazione della
Trinità. Scriveva André Grabar che "non si è mai giunti ad una iconografia soddisfacente dei dogmi trinitari: lo dimostra il fatto che tutti $i$ tentativi sono stati rapidamente abbandonati per essere sostituiti da altri, altrettanto discutibili e ugualmente effimeri" 47 ; in effetti, scorrendo le immagini trinitarie diffuse nel Medioevo è difficile trovarne una che assomigli all'esempio del Tolomeo marciano: un'unica figura tricefala, che troneggia sull'universo e abbraccia il cosmo, con al centro il Padre anziano e barbuto, affiancato da due volti giovanili e imberbi. L'immagine trinitaria era stata introdotta nell'area di Kastoria e Ochrida nella seconda metà del XIII secolo da artisti occidentali, probabilmente nell'ambito del Concilio di Lione e della polemica che ne seguì sull'Unione fra la chiesa di Roma e il patriarcato di Costantinopoli: esempi analoghi si trovano infatti nella chiesa di san Giorgio a Omorphoklesia, nei pressi di Kastoria (fig. 11), con il non trascurabile dettaglio che lo Spirito Santo è rappresentato dalla testa di una colomba ${ }^{48}$. Saška Bogevska ritiene che l'immagine del Tolomeo marciano si leghi appunto agli esempi di Omorphoklesia e del più antico affresco di Koubelidiki, nonostante non indaghi sulla trasformazione dell'iconografia dello Spirito Santo da colomba a testa giovanile. Una trasformazione che potrebbe anche avere la sua fonte nei codici del Breviari d'amor, come ad esempio nell'Escorial S.I. $3^{49}$, dove però la Trinità è raffigurata da una figura tricefala con i volti identici di tre anziani dalla barba lunga (fig. 12). Oltre al già citato argano che muove il mondo, i manoscritti del Breviari d'amor contenevano anche raffigurazioni delle sette donne delle Spirito Santo, e delle delle sette età del mondo ${ }^{50}$, e non sembra bizzarro ipotizzare che alla fine l'artista possa aver sfogliato uno di questi codici attraverso la mediazione di Andrea Teluntàs, oriundo occidentale e uomo di cultura $^{51}$ : miniatore e copista dovevano aver lavorato a stretto contatto, se anche in questo folio del codice marciano le immagini sono accompagnate da lunghe e articolate didascalie di mano di Teluntàs. Più in basso, nel primo cielo la Vergine in mandorla è affiancata da due angeli, e veglia sul secondo cielo, dove compaiono il sole e la luna e le stelle del firmamento, e sulla terra, con al centro il monte Sion, a destra una rocca fiorita che simboleggia il Paradiso

$\overline{47}$ A. GRABAR, Le vie della creazione nell'iconografia cristiana. Antichità e Medio Evo, Milano 1983, p. 141.

${ }^{48}$ L'immagine della testa alla sinistra del Padre è oggi molto rovinata e di difficile lettura. S. BOGEVSKA, The Holy Trinity in the Diocese of the Archbishopric of Ohrid in the second half of the $13^{\text {th }}$ Century, in Patrimonium 10, 2012, p. 154-157. Sull'iconografia trinitaria si vedano inoltre: M. ALPATOV, La Trinité dans l'art byzantin et l'icône de Rublev, in Echos d'Orient, 26-27, 1927-1928, p. 150-186; A. M. D’ACHILLE, Sull'iconografia trinitaria medievale: la Trinità del Santuario sul Monte Autore presso Vallepietra, in Arte Medievale, n.s., 5/1, 1991, p. 49-73

${ }^{49}$ C. MIRANDA GARCÍA-TEJEDOR, op. cit. (n. 15), p. 313-373.

${ }^{5}$ C. MIRANDA GARCÍA-TEJEDOR, Iconografia de las edades del mundo o de la Iglesia en el Breviario d'Amor de Mantfre Ermengaud de Bèzier, in Boletín de la Real Academia de la Historia, 191, 1994, p. 455-498.

${ }^{51}$ E. MIONI, op. cit. (n. 2), p. 58 


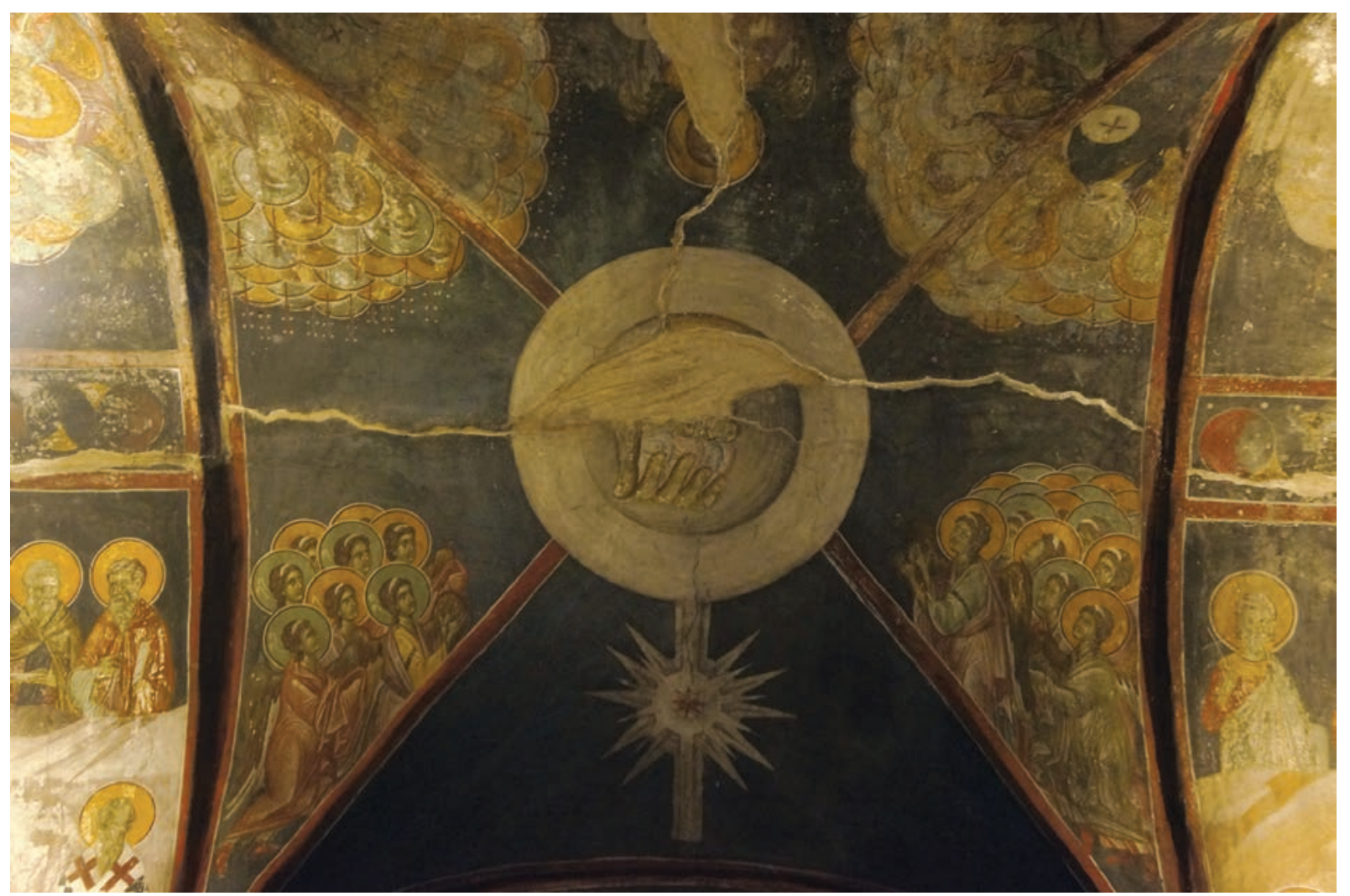

Fig. 13. Gračanica, monastero. Le anime dei giusti raccolte nella mano di Dio.

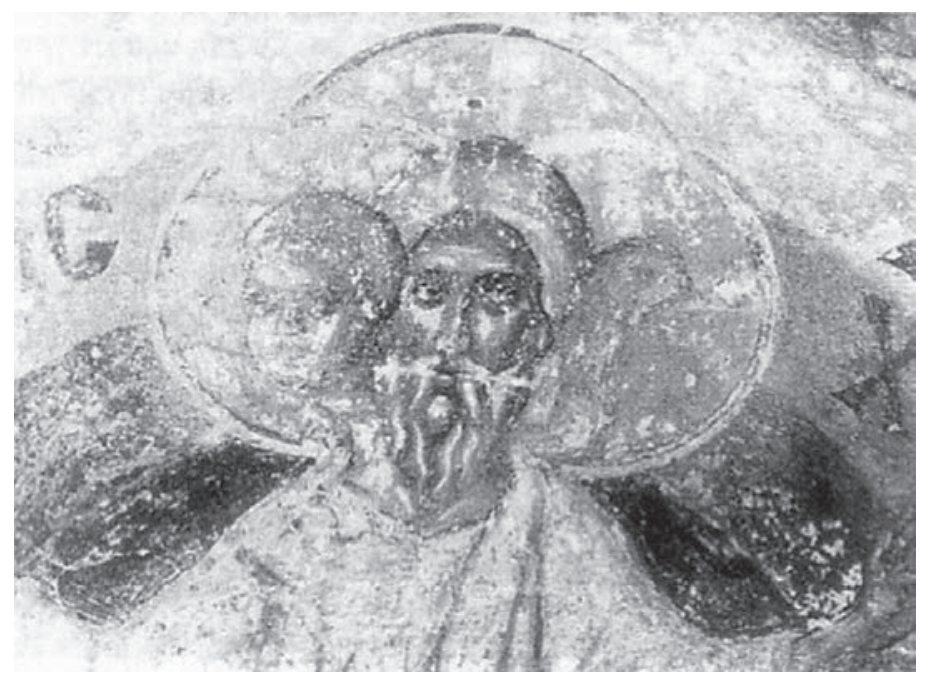

Fig. 11. Omorphoklesia, Chiesa di San Giorgio. Dettaglio della Trinità tricefala

e che sovrasta gli invalicabili confini di Gades e i suoi mari. L'intero orbis christianus è sorretto dalla mano di Dio: il singolare dettaglio ricorda la scena affrescata nell'esonartece della chiesa del monastero di Gračanica, fondazione del kral Milutin nel 1321, dove le anime dei giusti, un gruppo di bebè in fasce, sono accolti dalla mano di Dio che li sostiene (fig. 13), ripetuta qualche decennio più tardi nella chiesa dei Santi Apostoli a Tessalonica ${ }^{52}$.

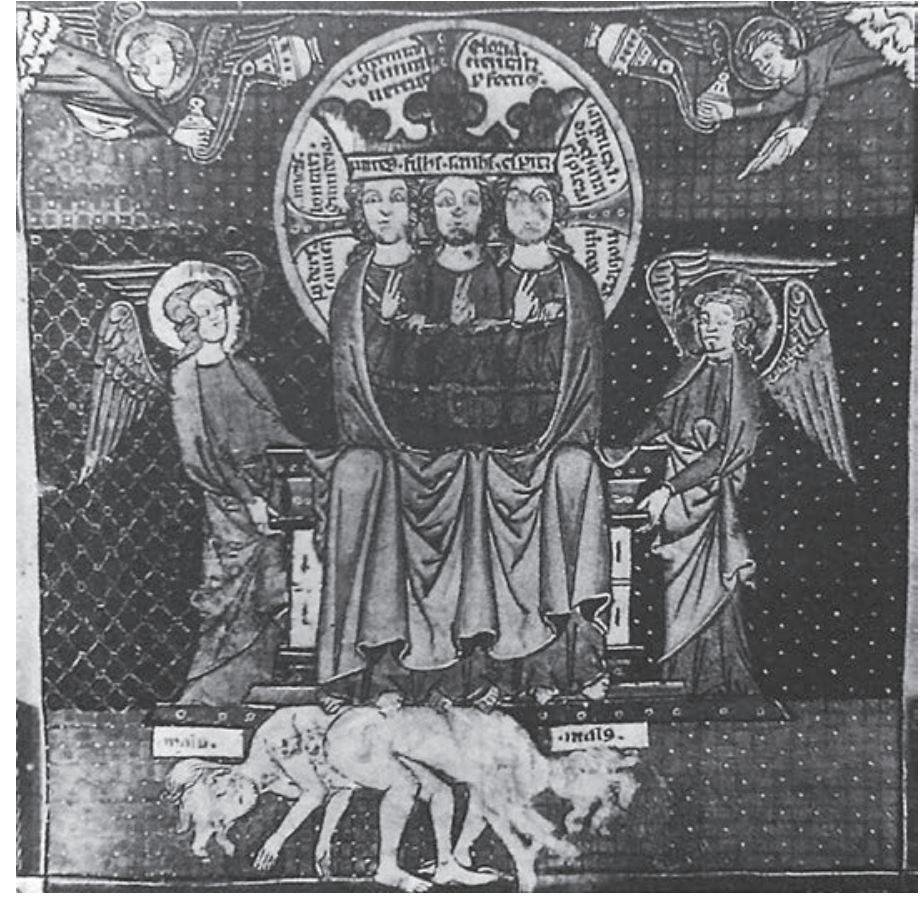

Fig. 12. El Escorial, Real Biblioteca del Monasterio de San Lorenzo, SI3, fol. 16r. Trinità tricefala.

Le didascalie di Teluntàs sono particolarmente dettagliate e prolisse, e proseguono sul folio successivo, sotto una rap-

${ }^{52}$ La decorazione fu realizzata dopo la deposizione nel 1314 del patriarca di Costantinopoli Nifone, che pure aveva promosso la fondazione dell'edificio come ricorda un'iscrizione dedicatoria; il programma infatti si deve a Paolo, egoumeno del monastero e discepolo di Nifone, indicato come "secondo fondatore". A. XYNGOPOULOS, Les fresques de l'Eglise des Saints-Apôtres à Thessalonique, in Art et société sous les Paléologues, Actes du Colloque organise par l'Association internationale des études byzantines à Venise en septembre 1968, Venezia 1971, p. 83-89; D. MOURIKI, Stylistic Trends in Monumental Painting of Greece at the Beginning of the Fourteenth Century, in D. MOURIKI, Studies in Late Byzantine Painting, London 1995, p. 9 (pubblicato per la prima volta in: L'art byzantin au debut du XIVe siècle, Belgrade 1978); M. RAUTMAN, Aspects of Monastic Patronage in Palaeologan Macedonia, in The Twilight of Byzantium. Aspects of Cultural and Religious History in the Late Byzantine Empire, S. ĆURČIĆ e D. MOURIKI (a cura di), Princeton 1991, pp. 71-72. Maria Parani ritiene che gli affreschi dell'esonartece furono realizzati fra il 1328 e il 1334: M. G. PARANI, Reconstructing the Reality of Images. Byzantine Material Culture and Religious Iconography (11th-15th Centuries), Leiden / Boston 2003, p. 266. 


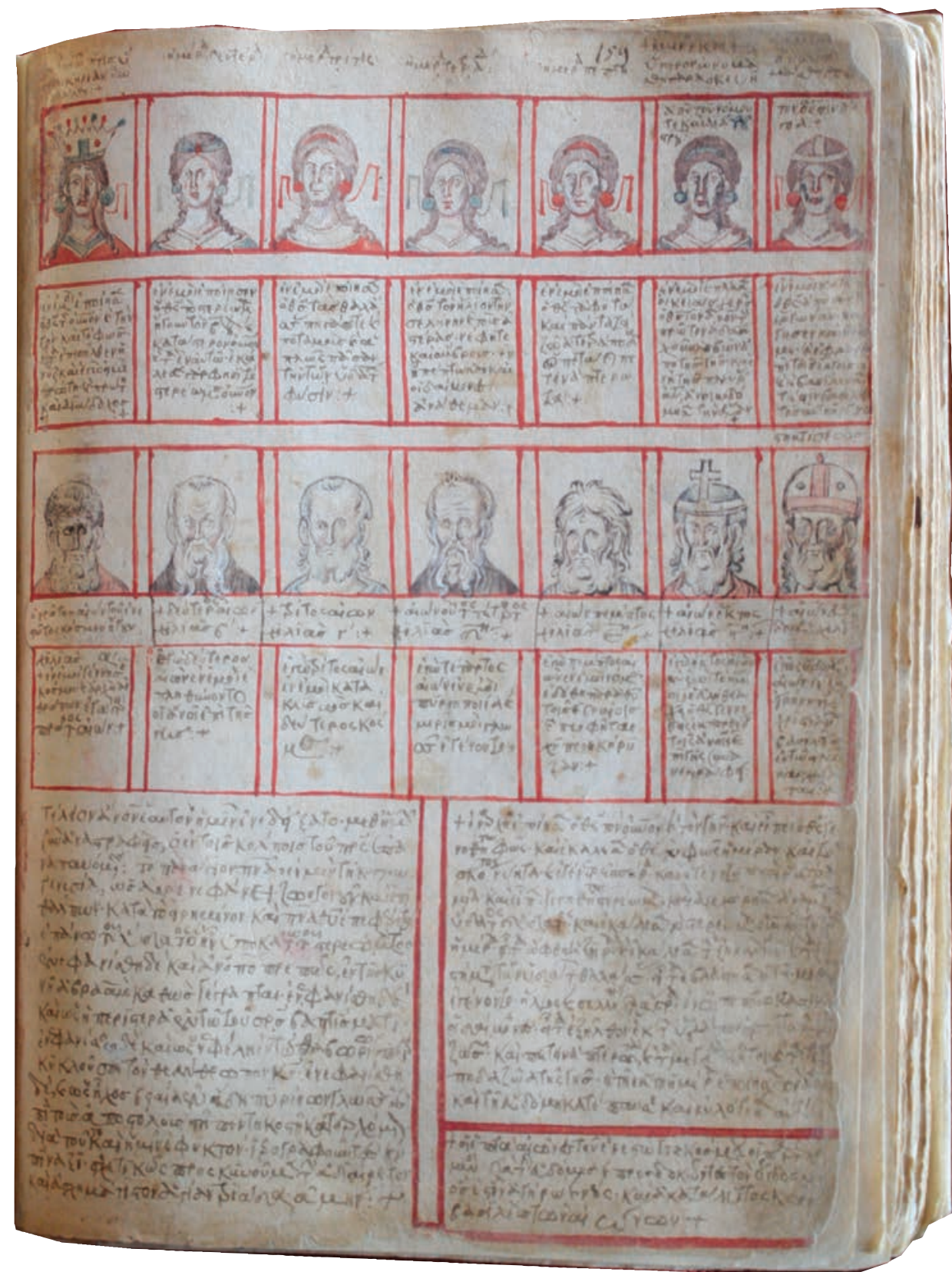

Fig. 14. Venezia, Biblioteca Nazionale Marciana, Marc. gr. Z. 516 (904), fol. 159r. I sette giorni della Creazione e i sette millenni della storia.

presentazione che riassume la nascita e la storia del mondo. L'artista dipinge i sette giorni della Creazione come sette volti di donna, ciascuno accompagnato nel riquadro sottostante dal corrispondente passo della Genesi; più in basso sette volti anziani e barbuti personificano invece i sette millenni della storia universale, anch'essi accompagnati da un riquadro che ne riassume gli eventi più importanti (fig. 14). Le fanciulle indossano degli orecchini, simili a quelli della Chatum al f. $2 \mathrm{~V}$, mentre le acconciature si diversificano, dalla corona del primo giorno ai più semplici cerchielli. Per quanto riguarda gli anziani, il disegno pone l'accento sul sesto e settimo millennio, e cioè quelli che corrispondono all'èra cristiana: il sesto indossa un copricapo aperto sul capo con una croce, mentre il settimo indossa una mitra vescovile, un'indicazione forse del passaggio da una fase più "eroica" e clandestina del cristianesimo a una più stabile e ufficiale nel corso dell'ultimo millennio.

La terza tavola (f. 159v, fig. 15) recupera un soggetto già svolto nel primo fascicolo, l'incessante volgere del tempo. $\mathrm{Al}$ centro di tutto è proprio il Tempo, ó

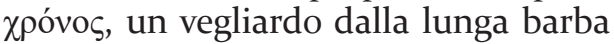
raffigurato a mezzo busto entro un clipeo; seguono poi altri tre centri concentrici, che rappresentano rispettivamente i dodici mesi, ritratti come volti di profilo, le fasi solari e lunari, e infine le settimane dell'anno. Fra queste, tre volti femminili e incoronati indicano la Pasqua, la domenica dopo la Pasqua e la Pentecoste. Ai lati dell'intero schema, le personificazioni delle quattro stagioni abbracciano il cosmo.

L'ultimo folio sorprende ancora una volta per il soggetto trattato: al centro della pagina quattro giovani si aggrappano l'uno alle gambe dell'altro; attorno quattro medaglioni sono agli angoli del folio, e altri quattro clipei più piccoli fra di essi, ai margini della carta (fig. 16). La didascalia scioglie l'intricato groviglio di corpi e teste: si tratta della rara raffigurazione della cosiddetta teoria degli umori, e ancora una volta si affonda nella cultura classica. La medicina è l'ultima delle discipline che ancora mancavano nelle illustrazioni del Marc. gr. Z. 516 (904): eppure ricopriva un ruolo di tale importanza nella Bisanzio paleologa che quando fra il 1332 e il 1335 Andronico III volle allearsi con il re di Napoli Roberto d'Angio, gli inviò in dono un codice con gli scritti di Galeno ${ }^{53}$. Elpidio Mioni suggeriva che la fonte

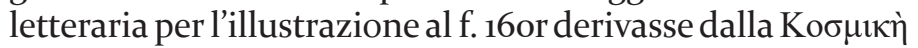

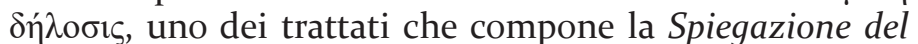
mondo scritta da Teodoro II Duca Lascaris alla metà del Duecento, che secondo quanto lo stesso niceno scriveva nel proemio a Giorgio Muzalone doveva contenere pressoché tutto: e Teodoro tratta diffusamente anche il concetto di temperamento e la teoria degli umori54. Ma il tono, e le argomentazioni di Teodoro sembrano distanti dallo spirito quasi illuminista che anima la vita intellettuale pochi decen-

\footnotetext{
${ }_{53}$ Nella scelta giocò sicuramente un ruolo fondamentale anche la passione di Roberto d'Angiò per i testi medici in generale, e per Galeno in particolare: il sovrano si fece tradurre da Niccolò di Reggio almeno ventisette opere di Galeno dal greco al latino. G. CAVALLO, La trasmissione scritta della cultura greca antica in Calabria e in Sicilia tra i secoli X-XV, in Scrittura e Civilta, 4, 1980, p. 233-235. Per una panoramica sulla medicina a Bisanzio: T. S. MILLER, The Birth of the Hospital in the Byzantine Empire, Baltimore 1985; G. CAVALLO, I libri di medicina: gli usi di un sapere, in Maladie et société à Byzance, E. Patlagean (dir.), Spoleto 1993, p. 43-56; P. BADENAS DE LA PENA, Byzantine Medical Book and the Diffusion of Byzantine Medicine in the Eastern Mediterranean. Introduction, in Medicina nei secoli, 11/3, 1999, p. 461-476; M.-H. CONGOURDEAU, La médecine à Nicée et sous les Paléologues: état de la question, in Philosophie et sciences à Byzance de 1204 à 1453. Les textes, les doctrines et leur transmission, Actes de la Table Ronde (Paris, 2001), M. Cacouros e M.-H. Congourdeau (dir.), Leuven / Paris / Dudley (MA) 2006, p. 185-188.

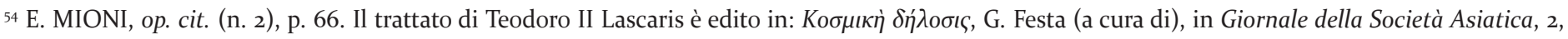
1898, p. 97-114.
} 


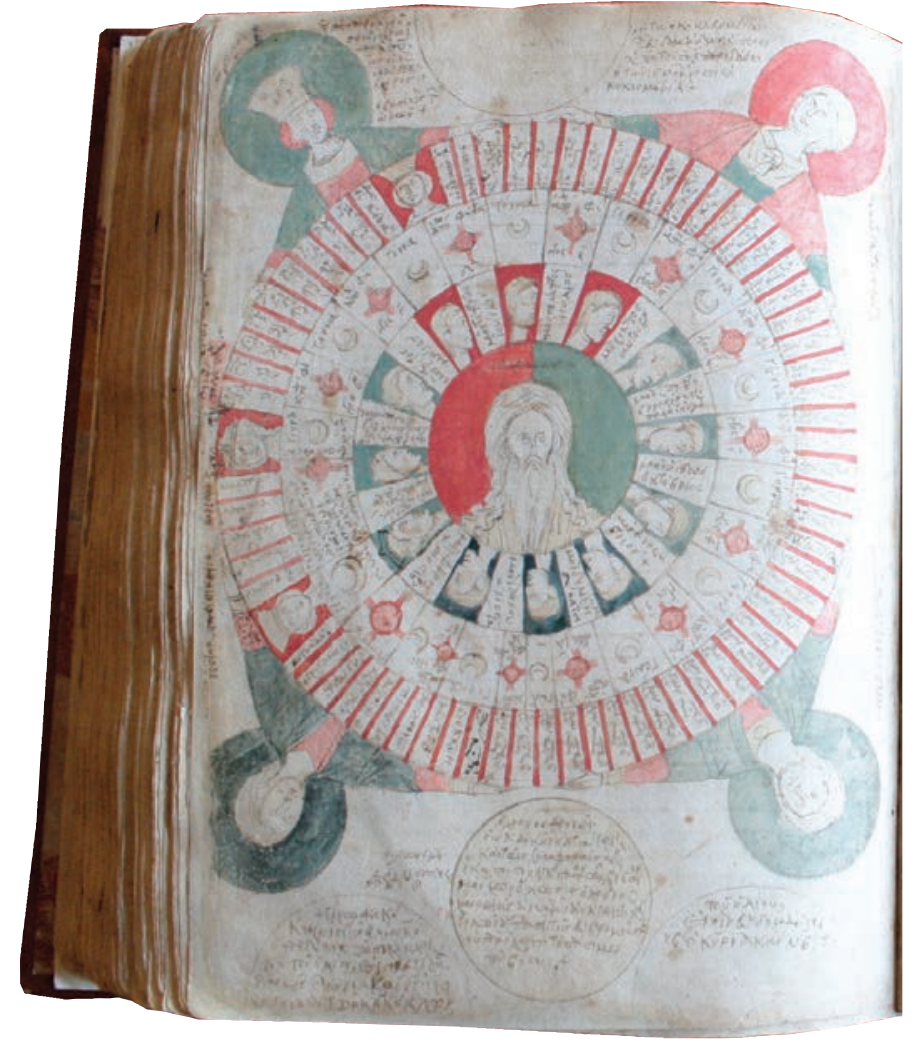

Fig. 15. Venezia, Biblioteca Nazionale Marciana, Marc. gr. Z. 516 (904), fol. $159 \mathrm{v}$. Il tempo e lo scorrere dell'anno.

ni più tardi: il suo indugiare su temi quali la volubilità della sorte, l'instabilità dell'esistenza umana, e il pessimismo che permea quest'opera e i più tardi Trattati morali 55 incarnano presupposti diversi da quelli già visti in atto nelle pagine del Tolomeo marciano. Anche per questo motivo sembra più opportuno suggerire una fonte più vicina alla vivacità culturale dei primi anni del XIV secolo, come ad esempio il

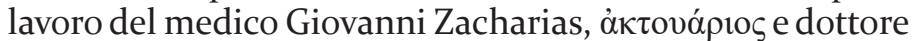
personale di Andronico III, che in gioventù scrisse un trattato dove ripropose la teoria degli umori, e esponendo i casi clinici che si era trovato ad affrontare illustrò la necessità di un'eziologia che non si limitasse ai soli sintomi fisiologici, ma indagasse anche la salute intellettuale del paziente ${ }^{56}$.

L'immagine è ad ogni modo la giusta chiosa alla cosmologia illustrata nei folii del Tolomeo marciano: proprio Galeno, che nel II secolo sviluppo gli studi di Ippocrate sulla teoria degli umori ${ }^{57}$, scriveva infatti che i medici, assieme a

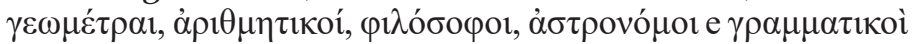
sono i più vicini a comprendere $\mathrm{Dio}^{58}$.

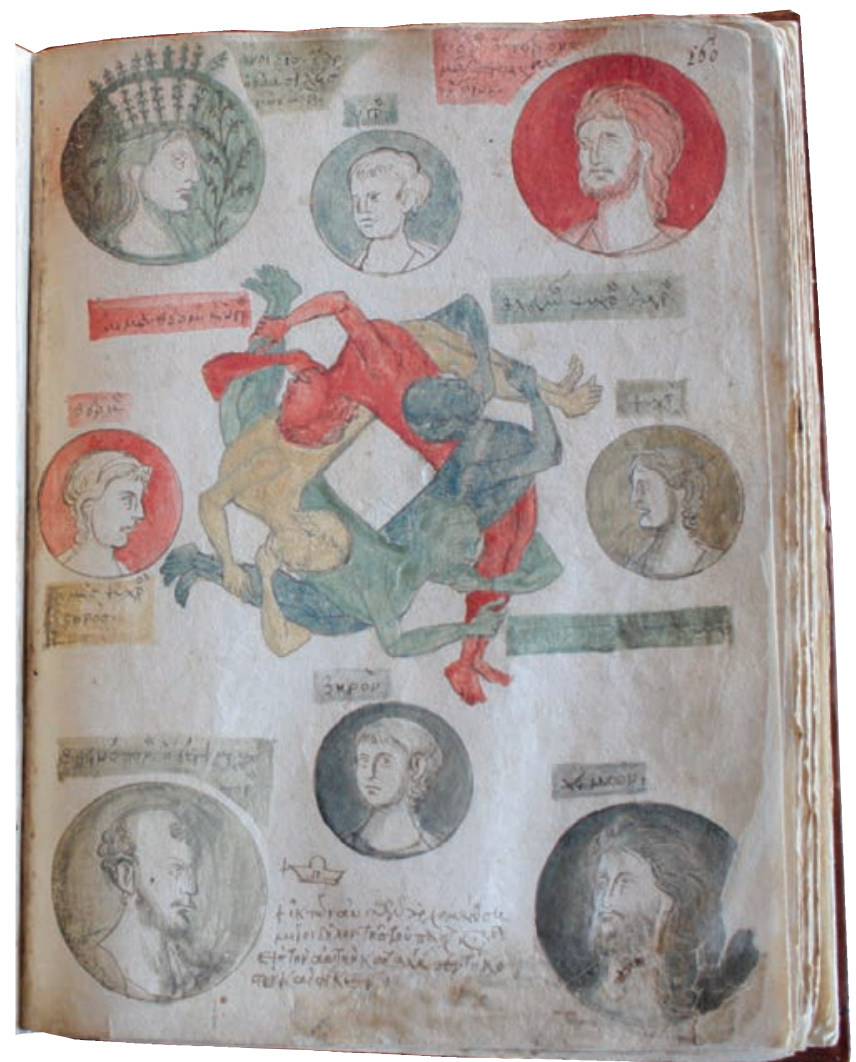

Fig. 16. Venezia, Biblioteca Nazionale Marciana, Marc. gr. Z. 516 (904), fol. 160r. Compenetrazione di materia e umori.

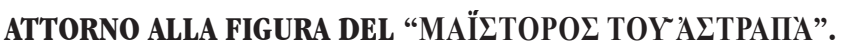

Le miniature appena descritte descrivono la visione del mondo che caratterizza l'umanesimo bizantino di età paleologa, in particolare fra la fine del XIII secolo e l'inizio del XIV: il tentativo intellettuale di integrare nell'ortodossia cristiana la tradizione scientifica classifica si sposa nel Marc. gr. Z. 516 (904) con un ciclo allegorico-filosofico, una sorta di enciclopedia illustrata di idee e teorie che circolavano fra le élites intellettuali di Costantinopoli e Tessalonica. Ma se Andrea Teluntàs si occupa di copiare i testi e stendere le didascalie dei fascicoli inseriti, e probabilmente di illustrare anche gli schieramenti militari della Tactica Theoria di Eliano e le macchine descritte nelle due opere di Erone di Alessandria59, a chi si devono le miniature dei fascicoli aggiunti?

$\mathrm{Al} \mathrm{f}$. Ir è apposta a grandi lettere purpuree una nota di

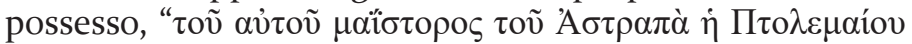
$\beta v ́ \beta \lambda$ o $\varsigma^{\prime}$, accanto ad un frammento da Euripide datato al XIV secolo $^{60}$. Nel domandarsi chi sia questo "maestro Astrapas"

55 D. G. ANGELOV, The Moral Pieces by Theodore II Laskaris, in Dumbarton Oaks Papers, 65-66, 2011-2012, p. 246.

${ }^{56} \mathrm{Fra}$ i casi che Zacharias descrive ce n'è uno che riguarda un uomo che soffre di disturbi al fegato e che infine si ammala di itterizia: l'indagine del giovane medico lo conduce a svelare la causa primigenia della malattia, un'ingiustizia che l'uomo aveva subito e che non era riuscito a superare. Un'ampia presentazione dei temi trattati nel De urinis, compresa l'ampio excursus di Zacharias sull'importanza degli studi filosofici per intraprendere la professione medica, in: A. HOHLWEG, John Actuarius' “De methodo medendi”- On the New Edition, in Dumbarton Oaks Papers 38, 1984, (= Symposium on Byzantine Medicine), p. 121-133.

57 Su Galeno, i suoi rapporti con la medicina di Ippocrate e la sua influenza sulla medicina a Bisanzio, si vedano: G. BAADER, Galen im mittelalterlichen Abendland, in Galen: Problems and Prospects, Y. NUTTON (a cura di), London 1981, p. 213-228; P. MORAUX, Galien de Pergame. Souvenirs d'un médecin, Paris 1985; A. TOUWAIDE, Une note sur la thériaque attribuée à Galien. Contribution à l'ètude de l'enseignement à Constantinople en XIVe et XVe siècles, in Byzantion, 67, 1997, p. 439-482.

${ }^{8}$ Citato in A. HOHLWEG, op. cit. (n. 56), p. 127.

${ }^{59} \mathrm{Al}$ di là delle immagini degli schieramenti militari, raffigurati come diagrammi e in quanto tali impossibili da attribuire a questa o a quella mano, un rapido sguardo alle figure umane tracciate in punta di penna lungo il testo dei Pneumatica e degli Automata di Erona mostrano una tale approssimazione e dilettantismo che risulta impossibile pensarli della stessa mano che illustrò le miniature dei fascicoli aggiunti.

${ }^{60}$ A. TURYN, The Byzantine Manuscript Tradition of the Tragedies of Euripides, Urbana (IL) 1957, p. 361. Mioni ne ha invece anticipato la scrittura agli ultimi decenni del XIII secolo: E. MIONI, op. cit. (n. 2), p. 59, n. 7 . 


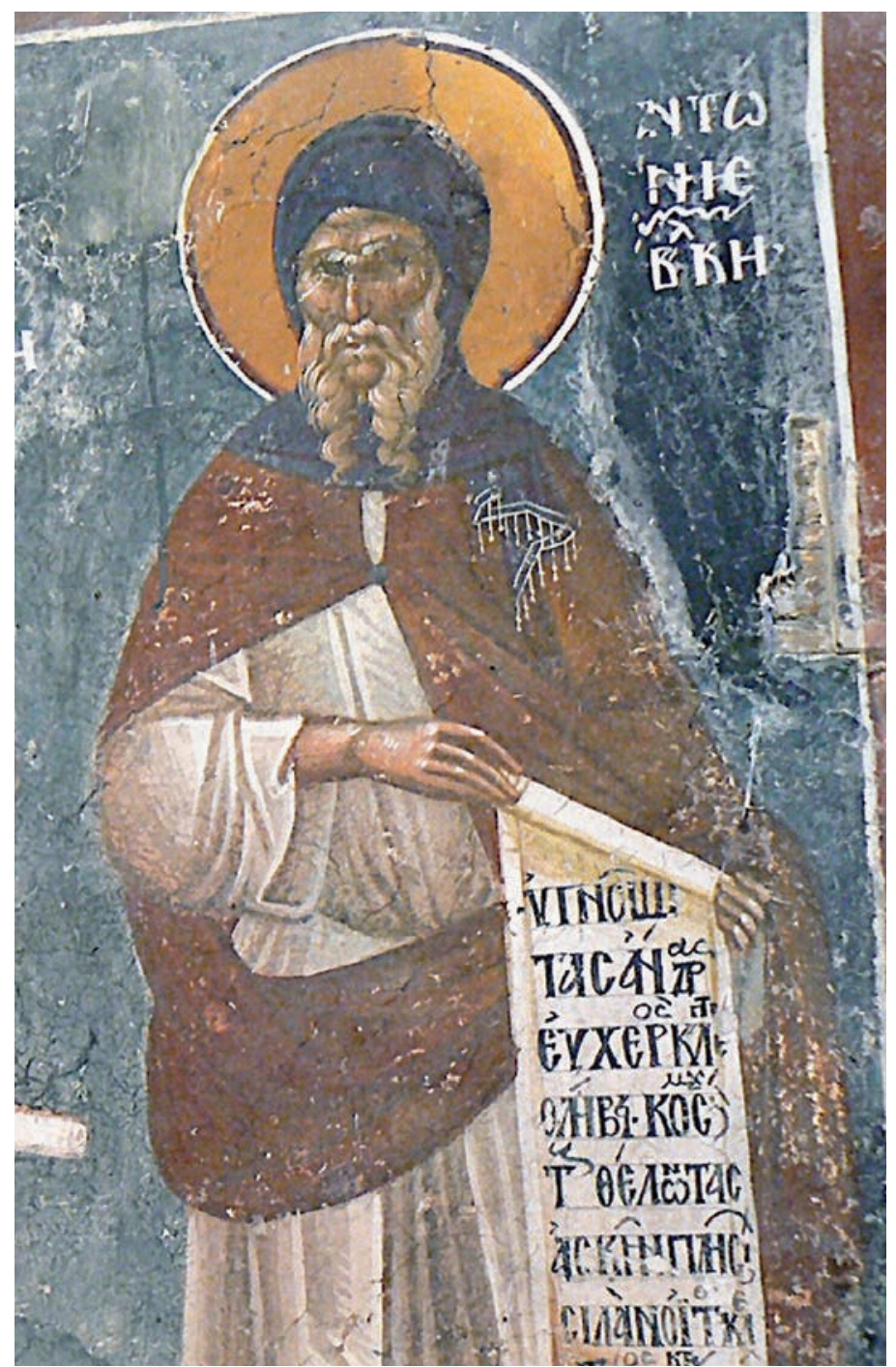

Fig. 17. Čučer, chiesa di San Niceta. Sant'Antonio.

il pensiero corre a Michele Astrapas, che insieme a Eutichio realizzò alcuni affreschi fra Tessalonica e la Serbia fra la fine del Duecento e l'inizio del Trecento ${ }^{61}$, nonostante non siano attestati altri codici da lui sottoscritti. La figura di Michele
Astrapas è sfuggente, eppure il suo nome è conosciuto da quasi un secolo: fu individuato da Louis Bréhier nello scudo di un santo guerriero affrescato nella chiesa di San Giorgio a Staro Nagoričino ${ }^{62}$, anche se poi l'associazione di Michele con l'appellativo Astrapas appare in un singolo caso, nel ciclo della Vergine Peribleptos a Ochrida, mentre altrove troviamo il solo nome Michele (a Staro Nagoričino appunto, e negli affreschi di San Niceta a Čučer) oppure il solo nome Astrapas (nella chiesa della Bogoridica Ljeviška, nei pressi di Prizren) ${ }^{63}$. Quest'incertezza lascia dunque aperta la possibilità che accanto al Michele Astrapas che appone il proprio nome a Ochrida, Staro Nagoričino e Čučer, operassero altri membri della famiglia: conosciamo ad esempio il nome di Giovanni Astrapas, che Demetrio Triclinio cita in un trattato astronomico del primo decennio del XIV secolo

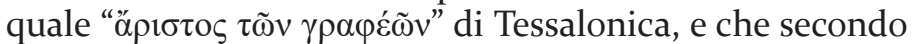
Kissas si sarebbe formato alla bottega di Eutichio e Michele collaborando alla Bogorodica Ljeviška ${ }^{64}$; Marković invece ipotizza che Eutichio sia il padre di Michele, istituendo quindi un ulteriore grado di parentela nella famiglia Astrapas ${ }^{65}$.

I confronti che è possibile istituire fra le immagini del Tolomeo marciano e le opere attribuite alla bottega Astrapas rivelano un'affinità, quasi che i disegni tracciati sul folio servissero da modello per le composizioni monumentali. Il tratto è rapido e sicuro - e qui torna in mente la suggestiva ipotesi di Lazarev, secondo cui l'appellativo $\alpha \sigma \tau p \alpha \pi \tilde{\alpha} \varsigma$ (folgore in greco) alludeva alla rapidità di esecuzione di Michele $^{66}$ - e la composizione si affida quasi esclusivamente alla punta del pennino, che delinea le espressioni dei volti, tratteggia il panneggio delle vesti, disegna barbe e capelli: al colore non resta che un ruolo di contorno, chiamato qua e là a esaltare dettagli (gli orecchini e le fasce fermacapelli delle personificazioni dei sette giorni della Creazione, fig. 14) oppure a connotare quasi scientificamente le immagini (i diagrammi armonici al f. 14or., come pure i quattro umori nella miniatura al f. 16or, fig. 16).

Sull'analisi stilistica grava naturalmente l'eccezionalità del manoscritto, le cui immagini partecipano alla definizione di questa cosmologia in un dialogo continuo e reiterato: alcuni dettagli possono però confortare nell'attribuzione del Marc. gr. Z. 516 (904) ad un artista della bottega Astrapas.

\footnotetext{
${ }^{{ }^{6}}$ Sull'attività della bottega di Michele Astrapas e di Eutichio si vedano fra gli altri: R. HAMAN-MACLEAN, Zu den Malerinschrifter der «Milutin- Schule», in Byzantinische Zeitschrift, 53, 1960, p. 112-117; P. MILIJKOVIC-PEPEK, L'oevre des peintres Michel et Eutych, Skopje 1967; B. TODIĆ, Protaton e la peinture serbe des premières décennies du XIV siècle, in L'art de Thessalonique et des pays balkaniques et les courants spirituels au XIVe siècle, D. Davidov (dir.), Belgrade 1987, p. 21-31.

${ }^{62}$ L. BRÉHIER, Les vieilles églises serbes. Impressions de voyage d'un congressiste, Nova Evropa 24/1, 1931, p. 12. Circa vent'anni prima Gabriel Millet aveva letto nella stessa chiesa il nome di Eutichio, dipinto sulla tunica di San Teodoro: G. MILLET, La derniére évolution de lsart byzantin, in Histoire de l'Art, depuis les premiers temps chrétiens jusquà nos jours, A. Michel (dir.), Paris 1908, III/2, p. 952.

${ }_{63}$ Per un riepilogo sulle questione delle firme, si veda M. MIHÁLNI, ad vocem Astrapas, Michele, in Enciclopedia dell'arte medievale, A.M. Romanini (dir.),

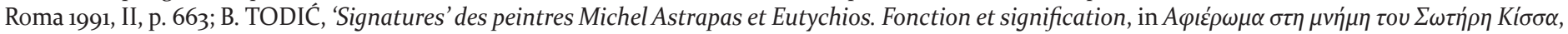
Thessaloniki 2001, pp. 643-662. Sul nome Astrapas nell'iscrizione dedicatoria della Bogoridica Ljeviška, Maria G. Parani ha ipotizzato possa riferirsi all'architetto della chiesa piuttosto che a un pittore: M.G. PARANI, op. cit. (n. 52), Leiden / Boston 2003, p. 262.

${ }^{64}$ Su Giovanni Astrapas: A. WASSERSTEIN, An Unpublished Treatise by Demetrius Triclinius on Lunar Theory, in Jahrbuch der Österreichischen Byzantinistik, 16, 1967, p. 154-155; S. KISSAS, Solunska umet nička porodica Astrapa [La famiglia degli artisti tessalonicesi Astrapas], Zograf, 5, 1974, p. 35-37; Prosopographisches Lexikon der Palaiologenzeit, E. Trapp (dir.), Wien 1976, I, p. 149-15o (nr. 1593); P. MILJKOVIC PEPEK, L’atelier artistique proéminent de la famille thessalonicienne d'Astrapas de la fin du XIIIe et des premiers décennies du XIVe siècle, Jahrbuch der Österreichischen Byzantinistik, 32,1982 (= XVI Internationaler Byzantinistenkongress, Akten 11/5, Wien 1981), p. 491-494; I. FURLAN, Scrittura e miniatura a Salonicco agli inizi del Trecento: una collaborazione tra Demetrio Triclinio e Giorgio Kalliergis?, in Miniatura. Lo squardo e la parola. Studi in onore di Giordana Mariani Canova, F. Toniolo e G. Toscano (dir.), Milano 2012, p. 86. Siamo inoltre a conoscenza di un Macario Astrapas, monaco nel monastero di Cortaite. Prosopographisches Lexikon der Palaiologenzeit, E. Trapp (dir.), Wien 1976, I, p. 150 (nr. 1594).

${ }_{65}$ Contestualmente, Marković ha rovesciato anche la prospettiva che vedeva la figura di Michele prevalere su Eutichio, attribuendo a quest'ultimo il ruolo di protomagistron nel ciclo della Vergine Peribleptos a Ochrida: M. MARKOVIĆ, The Painter Eutychios - Father of Michael Astrapas and Protomaster of the Frescoes in the Church of the Virgin Peribleptos in Ohrid, Zbornik Matice srpske za likovnu umetnost, 38, 2010, p. 9-34.

${ }^{66}$ V. LAZAREV, Storia della pittura bizantina, Torino 1967, p. 343, n. 121.
} 


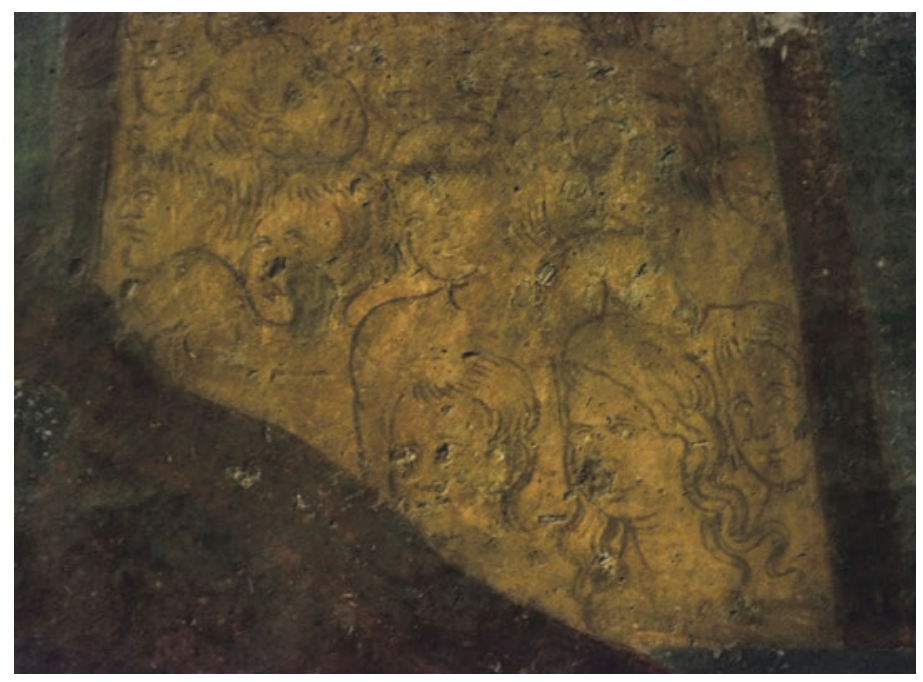

Fig. 18. Gračanica, monastero. Volti giovanili.

I tipi fisiognomici del manoscritto attingono allo stesso repertorio di volti che affollano i cicli di Staro Nagoričino, Čučer e della Bogorodica Ljeviška a Prizren: i visi eleganti, i nasi allungati e curvati ${ }^{67}$, gli occhi sgranati, le stesse barbe arricciate "french fork" ${ }^{68}$ o lunghe e a punta. Si prenda a confronto l'immagine del secondo millennio al f. 159r con il ritratto di Sant'Antonio a Čučer (fig. 17): in entrambe l'artista si serve di leggerissimi tratti per modellare le guance e le righe sulla fronte, e lo stesso sguardo laterale si tramuta in un'espressione quasi sinistra, corrucciata, a causa delle sopracciglia eccessivamente arcuate.

Ma è in un altra fondazione del kral Milutin, che nonostante l'assenza della firma di Astrapas è già stato attribuito alla medesima bottega ${ }^{69}$, che questa abilità ritrattistica è sublimata: si tratta della chiesa del monastero di Gračanica, ultima fondazione di Milutin databile al 1321. Nel ricchissimo ciclo iconografico sopravvive un pannello che non fu mai dipinto, e che dunque può offrire un importante termine di paragone con i disegni del manoscritto veneziano (fig. 18). Nella scena si affollano una serie di volti in primo piano, di tre quarti o di profilo, e compaiono alcuni stilemi tipici dell'artista del Tolomeo marciano, come per esempio nei ritratti entro clipeo che coronano la raffigurazione della teoria degli umori. La "frangetta" dei volti giovanili definita attraverso una serie di tanti tratti verticali, il profilo del naso pronunciato, "alla greca", nelle teste di profilo, il piccolo segno che modella il labbro inferiore, rendendo la bocca tumida - un dettaglio, questo, che nella serie dei Sette giorni della Creazione è amplificato dall'utilizzo del colore rosso, e che per esempio troviamo anche negli affreschi della Bogorodica Ljeviška.

Un'altra scena aiuta a comprendere meglio il rapporto fra il ciclo di Gračanica e il Marc. gr. Z. 516 (904), e cioè l'incoronazione di Simonida, moglie di Milutin e figlia di Andronico II (fig. 19): la sovrana porta una corona quasi identica a quella indossata dal primo giorno della Creazione

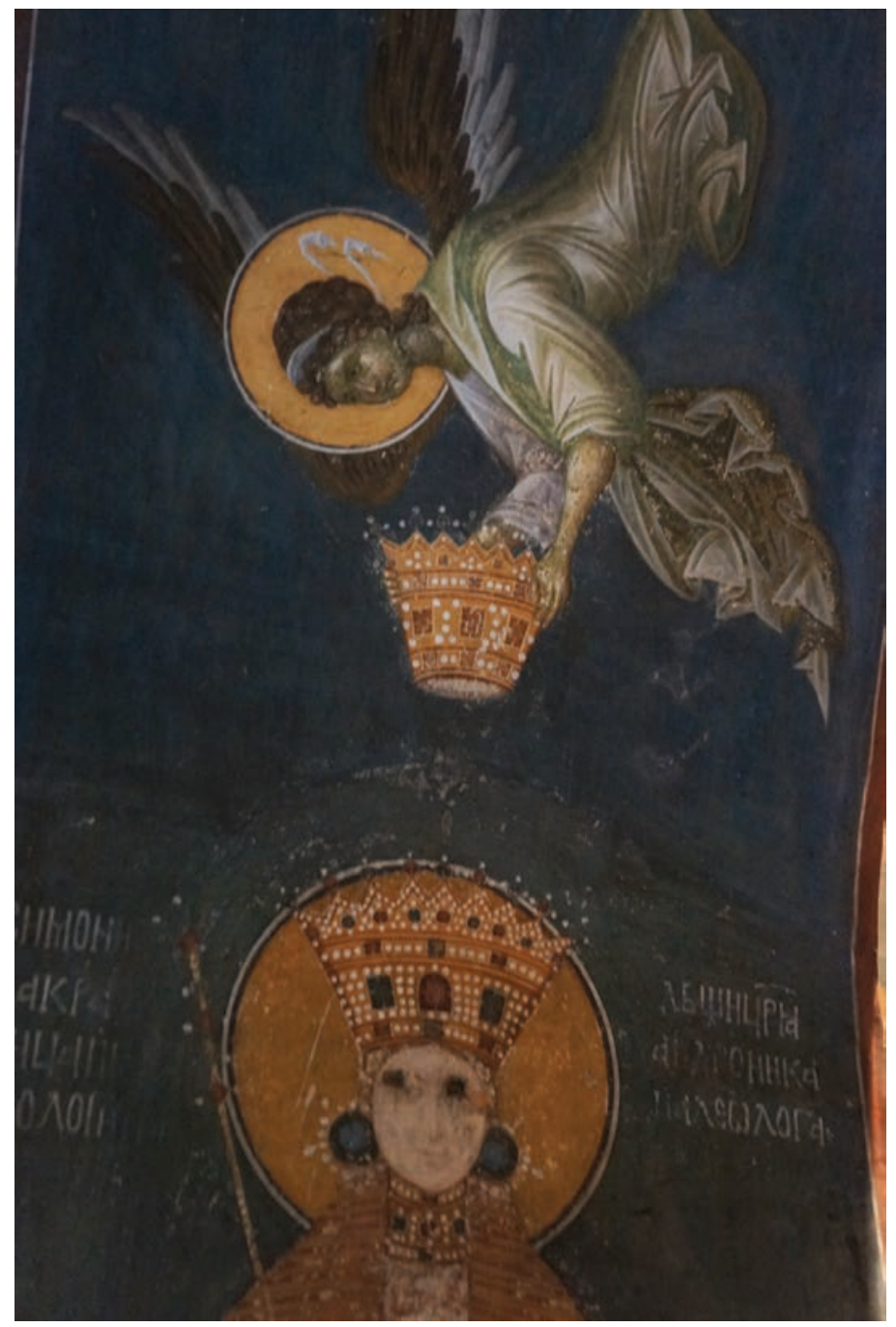

Fig. 19. Gračanica, monastero. Incoronazione della regina Simonida.

al f. 159r (fig. 20), con un rubino rosso al centro affiancato da due smeraldi (nel manoscritto l'immagine è semplificata, e l'ordine delle pietre invertito), e le pietre preziose alternativamente verdi e rosse dipinte sulle punte, mentre l'angelo in volo che tiene fra le mani una seconda corona mostra la stessa alternanza di penne marroni e penne bianche sulle ali come nell'immagine dei due angeli che affiancano la Vergine benedicente al 158v (fig. 10).

Le affinità fra le pitture di Gračanica e le immagini del Tolomeo marciano sono da attribuirsi, a nostro avviso, ad un medesimo atelier, che ruota attorno alle figure di Eutichio e Michele Astrapas, ma con il quale collaborò probabilmente anche Giovanni, e forse qualche altro membro della famiglia Astrapas di cui non si conserva il nome; e i due lavori furono eseguiti con ogni probabilità nello stesso volgere d'anni, dunque attorno al 1320. La leggerezza delle composizioni nel manoscritto veneziano vira verso un'eleganza raccolta e misurata, che si incontrano nelle opere della bottega degli Astrapas a partire dalla fine del primo decennio del Trecento,

\footnotetext{
${ }_{67}$ Doula Mouriki usa l'espressione "drooping nose", e su questo dettaglio costruisce la sua ipotesi di attribuzione alla bottega di Michele Astrapas e Eutichio anche degli affreschi di San Nicola Orphanos a Tessalonica. D. MOURIKI, op. cit. (n. 52), 6-7. L’ipotesi di Mouriki si serve inoltre degli studi di Radojčić, secondo cui San Nicola Orphanos è una delle tre chiese di Tessalonica decorate su iniziativa del kral Stefano Uroš II Milutin. S. RADOJČIĆ, Staro srpsko slikarstvo, Belgrado 1966, p. 87.

${ }^{68} \mathrm{E}$ cioè che termina con due punte, come nel caso del riquadro con il quarto millennio al f. 159r. Ho trovato la definizione di questo tipo barba qui: [http:// beardoholic.com/best-beard-styles].

${ }^{69}$ S. KISSAS, op. cit. (n. 64), p. 37.

${ }^{70}$ B. TODIĆ, Serbian Medieval Painting: The Age of King Milutin, Belgrade 1999.
} 


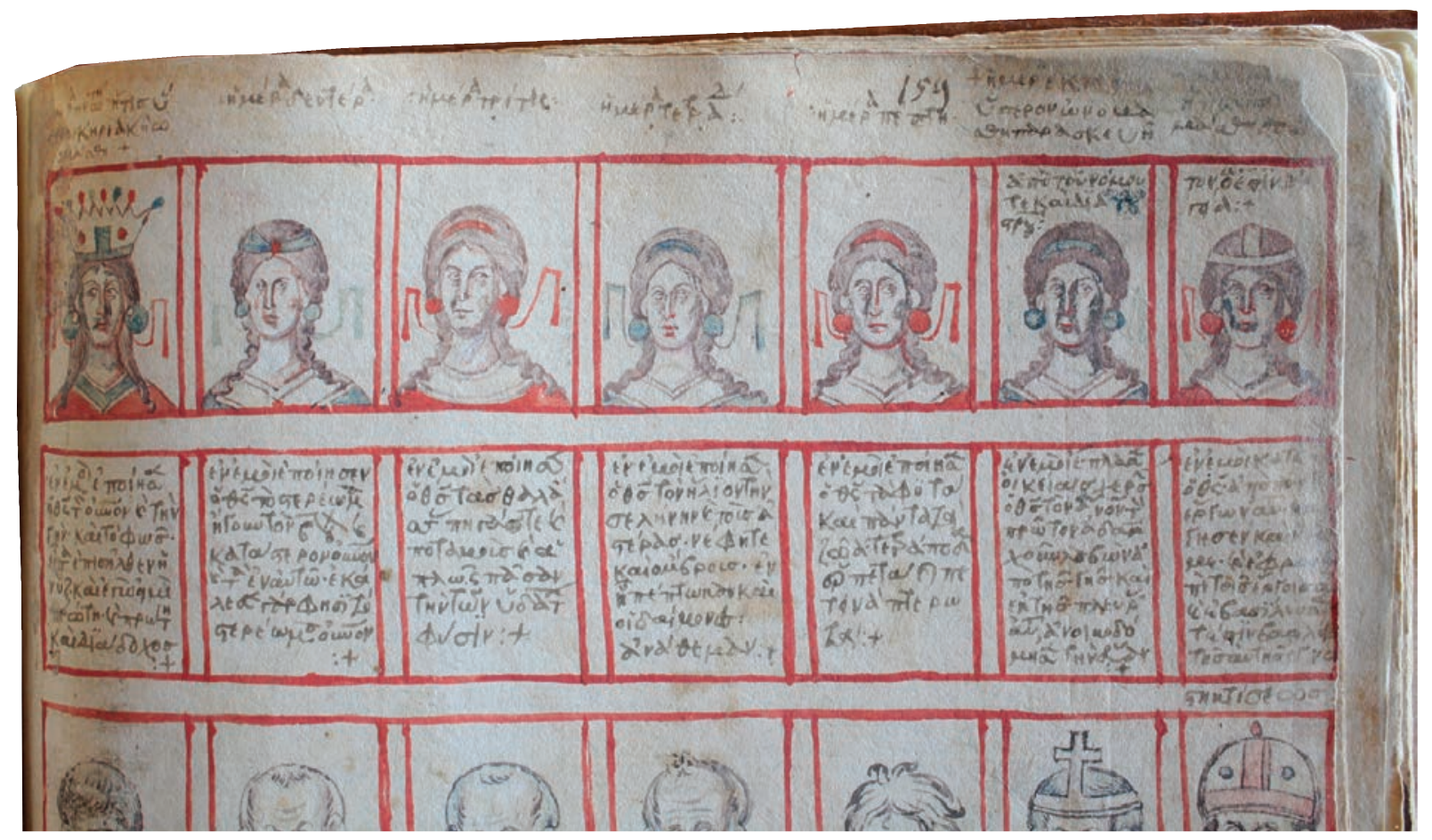

Fig. 20. Venezia, Biblioteca Nazionale Marciana, Marc. gr. Z. 516 (904), fol. 159r. Particolare del primo giorno della Creazione.

un'evoluzione che avviene più o meno contemporaneamente all'inizio del loro rapporto con il kral Stefano Uroš II Milu$\operatorname{tin}^{70}$, e potrebbe essere stata suggerita proprio dal sovrano serbo, desideroso di confrontarsi con i più aggiornati risultati artistici à la mode di Costantinopoli ${ }^{71}$.

\section{CONCLUSIONI.}

Se, dopo l'analisi stilistica, riprendiamo in mano i dati codicologici descritti all'inizio di questo testo, ne deriva un'interpretazione quantomai univoca: Andrea Teluntàs copia il manoscritto utilizzando delle carte che mostrano filigrane diffuse a Genova e a Dubrovnik sul finire degli anni Dieci, collaborando fianco a fianco con un artista, probabilmente lo stesso "maestro Astrapas" che appone la sua nota di possesso al f. ir, che potrebbe essere Michele oppure un altro parente formatosi nella bottega di famiglia. La collaborazione fra un oriundo occidentale di Nauplia, amante della cultura ma con una conoscenza non impeccabile della lingua greca, e un artista greco che lavorò al servizio del kral serbo Stefano Uroš II Milutin in Serbia e a Tessalonica, non poté che avvenire proprio nella città portuale della Macedonia. La fortunata joint-venture si servì del meglio che le due culture potessero offrire, approfittando di una congiuntura politica e intellettuale favorevole: le carte acquistate da un mercante latino, le suggestioni iconografiche di probabile origine occidentale, mediate probabilmente da esempi mo- numentali "ibridi" come a Omorphoklesia, oppure suggerite dallo stesso Teluntàs attraverso dei manoscritti d'outremer, il fervore intellettuale che anima i circoli cittadini, dove si discutono e si scrivono trattati di scienza e di filosofia che reinterpretano gli antichi maestri. Tessalonica nel Trecento era una città che usciva dal cono d'ombra di Costantinopoli,

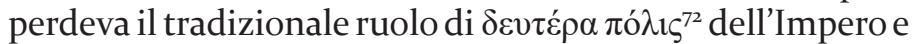
si affermava come centro politico e culturale di prim'ordine: anche alcuni membri della famiglia imperiale spostarono lì la propria residenza, da Irene del Monferrato, moglie di Andronico II, che vi rimase dal 1303 fino alla morte avvenuta nel 1317, ai figli dello stesso imperatore, Michele IX e Demetrio, che governarono la città negli anni venti, fino ad Anna Paleologina, moglie di Andronico III, che preferiva Tessalonica alla capitale ${ }^{73}$. Ed è curioso che anche il Marc. gr. Z. 516 (904) partecipi alla "rinascenza" cittadina attingendo ad autori che godevano di credito e ammirazione a Tessalonica. Si prenda ad esempio Niceforo Cumno: sebbene Tinnefeld abbia escluso che le sue lettere fossero oggetto di letture collettive nei circoli intellettuali di Tessalonica ${ }^{74}$, il legame di Cumno con la città macedone fu senza dubbio intenso. L'imperatore lo nominò governatore nel 1309, con la città in difficoltà dopo aver fronteggiato la minaccia catalana, e Cumno vi restò non più di un anno, dimostrandosi comunque un ottimo amministratore. E lo stesso Cumno dovette rimanere legato alla città, se poco dopo il rientro a Costantinopoli si premurò di inviare agli amici più stretti rimasti a Tessalonica un lungo

${ }^{71}$ T. GOUMA-PETERSON, The Frescoes of St. Euthymios in Thessaloniki: Patrons, Workshop and Style, in S. Curčić e D. Mouriki (dir.), op. cit. (n. 52), p. 126. Sul ruolo dei committenti anche nella definizione dello stile paleologo si vedano inoltre: H. BELTING, Das Illuminierte Buch in der spätbyzantinische Gesellschaft, Heidelberg 1970; H. BUCHTAL e H. BELTING, Patronage in Thirteenth-Century Constantinople. An Atelier of Late Byzantine Book Illumination and Calligraphy, (Dumbarton Oaks Studies. 16), Washington 1978.

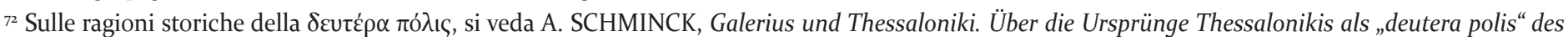
byzantinischen Reiches, in Byzantine Law, Proceedings of the International Symposium of Jurists (Thessaloniki, 10-13 December 1998), C. Papastathis (dir.), Thessaloniki 2001, pp. 117-133.

${ }_{73}$ D. BIANCONI, Tessalonica nell'età dei Paleologhi. Le pratiche intellettuali nel riflesso della cultura scritta, Paris 2005, p. 52. Su Anna Paleologina, si veda anche M. DELLA VALLE, Costantinopoli e Tessalonica al tempo di Anna Paleologina, in A. Iacobini e M. Della Valle (dir.), op. cit. (n. 2), p. 125-142.

${ }^{74}$ F. TINNEFELD, Intellectuals in Late Byzantine Thessalonike, in Dumbarton Oaks Papers, 57, 2003, , p. 158. 


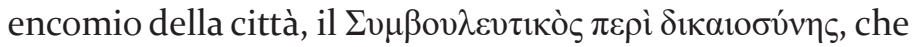
invitava i cittadini a superare le tensioni interne, toccando argomenti concreti come la disperazione delle classi meno abbienti e la corruzione dilagante ${ }^{75}$. Anche Giovanni Zacharias aveva progettato di trasferirsi a Tessalonica al principio del Trecento, nonostante i prolungati inviti a ripensarci da parte di Giorgio Lakapenos, secondo cui l'amico avrebbe rimpianto gli agi di Costantinopoli nel caotico disordine di una città portuale ${ }^{76}$.
Una città certamente caotica, al centro dei commerci fra Oriente e Occidente ${ }^{77}$, forse pericolosa come solo le città portuali sanno esserlo, ma dai gusti raffinati, ancora "cosi adornata ad arte e cosi superba per lo splendore dei suoi edifici" come qualche secolo prima scriveva Giovanni

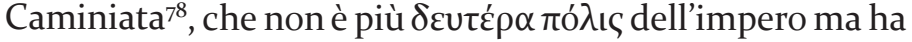
finalmente colmato il secolare scarto culturale fra Costantinopoli e le sue provincie.

\footnotetext{
75 La prima parte dell'encomio, tuttavia, contiene una breve ekphrasis della città, descritta a partire dalle bellezze delle aree circostanti (i boschi e i fiumi a occidente, i laghi e i campi fertili a oriente, il mare) fino alla perfezione delle chiese e dei palazzi.J. F. BOISSONADE, op. cit. (n. 19), p. 137; J. VERPEAUX, op. cit. (n. 14), p. 49-55; F. TINNEFELD, op. cit. (n. 74), p. 165.

${ }^{76}$ L'intera vicenda è descritta in una lettera di Lakapenos inviata nel 1299 a Zacharias: A. HOHLWEG, op. cit. (n. 56), p. 122.

77 A. LAIOU, Thessaloniki and Macedonia in the Byzantine Period, in Byzantine Macedonia: Identity, Image and History, Papers from the Melbourne Conference (July 1995), a cura di J. Burke e R. Scott, Melbourne 2000, p. 2.

${ }^{78}$ L'ekphrasis di Caminiata è del 904. La citazione in: D. BIANCONI, op. cit. (n. 73), p. 20.

* Ringrazio la professoressa Claudia Barsanti e Paolo Di Simone, per i loro suggerimenti durante la discussione seguita alla mia relazione dello scorso maggio a Parenzo. Un grazie particolare a Valentina Cantone, con la quale ho chiacchierato ripetutamente (e ancora continuo a farlo) sul Tolomeo marciano.
} 